\title{
Algebraic evaluation of matrix elements in the Laguerre function basis
}

\author{
A. E. McCoy and M. A. Caprio \\ Department of Physics, University of Notre Dame, \\ Notre Dame, Indiana 46556-5670, USA
}

(Dated: September 25, 2018)

\begin{abstract}
The Laguerre functions constitute one of the fundamental basis sets for calculations in atomic and molecular electron-structure theory, with applications in hadronic and nuclear theory as well. While similar in form to the Coulomb bound-state eigenfunctions (from the Schrödinger eigenproblem) or the Coulomb-Sturmian functions (from a related Sturm-Liouville problem), the Laguerre functions, unlike these former functions, constitute a complete, discrete, orthonormal set for square-integrable functions in three dimensions. We construct the $\mathrm{SU}(1,1) \times \mathrm{SO}(3)$ dynamical algebra for the Laguerre functions and apply the ideas of factorization (or supersymmetric quantum mechanics) to derive shift operators for these functions. We use the resulting algebraic framework to derive analytic expressions for matrix elements of several basic radial operators (involving powers of the radial coordinate and radial derivative) in the Laguerre function basis. We illustrate how matrix elements for more general spherical tensor operators in three dimensional space, such as the gradient, may then be constructed from these radial matrix elements.
\end{abstract}

\section{INTRODUCTION}

The Laguerre functions [1-3] constitute one of the fundamental basis sets for calculations in atomic and molecular electron-structure theory [1, 4] 6], with application in hadronic [712 and nuclear structure [13 15] theory as well. The Laguerre functions share the same functional form as the Coulomb bound-state wave functions (obtained as solutions to the Schrödinger equation central force eigenproblem) or the Coulomb-Sturmian functions (obtained as solutions to a related Sturm-Liouville problem). However, unlike these functions, the Laguerre functions constitute a complete, discrete, orthonormal set of square-integrable 
functions in three dimensions.

Numerical solution of quantum mechanical problems - for the present discussion, we can take this to mean one-body and many-body Schrödinger equation problems, although the applicability is more general - may be represented in terms of an expansion of the wave function on a set of basis functions. The Schrödinger equation eigenproblem, when expanded in a discrete basis, is recast as a Hamiltonian matrix eigenproblem [16]. To proceed with this approach, it is necessary to be able to evaluate the matrix elements of the operators of the problem, taken with respect to the expansion basis. Relevant operators may include those contributing to the Hamiltonian, transition operators, and operators representing moments or other static observables.

For numerical solution of many-body problems, the many-body basis functions are obtained as products of single-particle basis functions (more precisely, either symmetrized or antisymmetrized products, according to the statistics of the problem [17]). When the manybody problem is rotationally invariant, it is natural to consider an expansion in terms of single-particle basis functions which are themselves obtained as solutions to a rotationallyinvariant central force problem, for instance, the three-dimensional harmonic oscillator functions [18]. The solution sets to central force problems are well known to factorize into radial and angular functions, arising from the factorizability of the Hilbert space of squareintegrable functions on $\mathbb{R}^{3}$ as $\mathcal{L}^{2}\left(\mathbb{R}^{3}\right)=\mathcal{L}^{2}\left(\mathbb{R}^{+}\right) \times \mathcal{L}^{2}\left(S^{2}\right)$. The angular functions, on the sphere $S^{2}$, are the well-known spherical harmonics [19]. For each choice of angular momentum $l$, the associated radial functions form a complete set of square-integrable functions on $\mathbb{R}^{+}$. Matrix elements of spherical tensor operators (including rotationally-invariant scalar operators and vector operators) may be simplified through a corresponding factorization into radial and angular dependences, together with the Wigner-Eckart theorem [19] for the angular dependence.

Most basic among the central force problems are those for the harmonic oscillator potential and the Coulomb (hydrogenic) potential. Both these problems have a rich group theoretical structure (reviewed in Ref. [20]), which allows analytic expressions to be obtained for matrix elements of many operators of interest, built from the coordinate vector $\mathbf{r}$ and momentum vector $\mathbf{p}=-i \hbar \nabla$.

Underlying the convenience of these bases is the presence of an $\mathrm{SU}(1,1) \times \mathrm{SO}(3)$ dynamical group [20], which reflects the factorization of the problem into radial and angular coordi- 


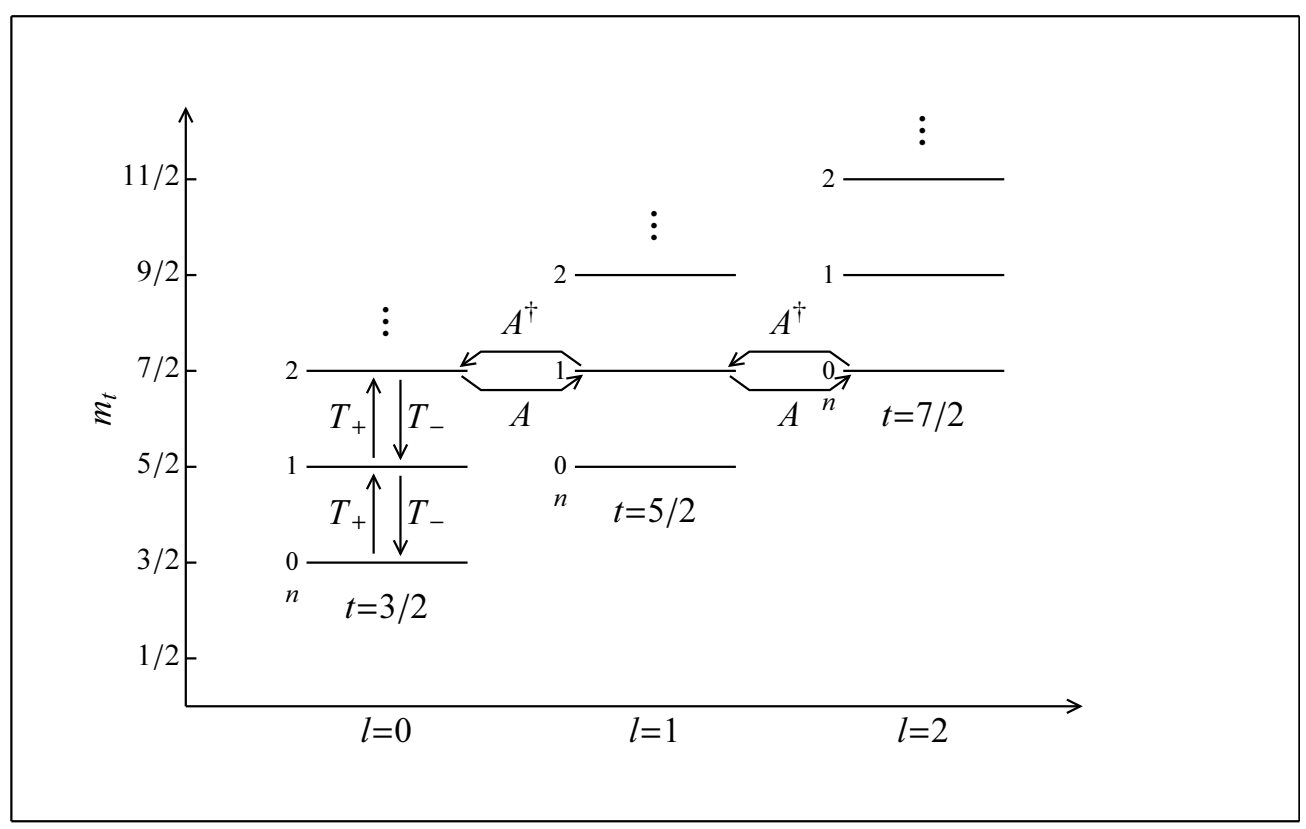

FIG. 1: Algebraic classification of basis functions, with respect to the $\mathrm{SU}(1,1) \times \mathrm{SO}(3)$ dynamical algebra, and the pattern of laddering operators $T_{ \pm}$and shift operators, $A^{\dagger}$ and $A$, connecting these functions. The quantum number $l$ labels the $\mathrm{SO}(3)$ irrep, and the dual quantum number $t$ labels the $\mathrm{SU}(1,1)$ irrep by its lowest weight, where $m_{t}$ is the $\mathrm{SU}(1,1)$ weight (see text).

nates - with $\mathrm{SU}(1,1)$ operating on the radial functions and $\mathrm{SO}(3)$ on the angular functions. The $\mathrm{SU}(1,1)$ spectrum generating algebra relates radial wave functions with different radial quantum number (node number) $n$, at the same angular momentum $l$. This $\mathrm{SU}(1,1)$ algebra has been widely studied for both the oscillator [21 23] and Coulomb [21, 22, 24 27] problems. The $\mathrm{SO}(3)$ algebra, in turn, is the standard angular momentum algebra, which relates angular wave functions (spherical harmonics) with different angular momentum projection quantum number $m$, at given $l[19]$.

While the $\mathrm{SU}(1,1) \times \mathrm{SO}(3)$ algebra thus provides the framework for relating states of the same angular momentum (and, in fact, for calculating matrix elements among these states, at least for certain suitable operators), an additional structure is required to connect basis states of different angular momentum. This connection is provided either by the factorization method (first applied by Schrödinger for the Coulomb problem [28] and subsequently generalized by Infeld and Hull [29]) or, equivalently, through the ideas of supersymmetric quantum mechanics (SUSY QM) [30]. 
More concretely, the relations among basis states provided by the algebraic structures are indicated in Fig. 1. To each angular momentum $l$ is associated a set of radial wave functions. These constitute a positive-discrete, infinite-dimensional irreducible representation (irrep) of $\mathrm{SU}(1,1)$. Radial wave functions differing in radial quantum number $n$ by \pm 1 , at fixed $l$, are related by the $\mathrm{SU}(1,1)$ ladder operators $T_{ \pm}$. These change the eigenvalue of the weight operator $T_{0}$, i.e., the weight $m_{t}=n+l+3 / 2$, by \pm 1 . Each $\mathrm{SU}(1,1)$ irrep is labeled by its lowest weight $t=l+3 / 2$. Thus, to each irrep (with label $l$ ) of the angular algebra $\mathrm{SO}(3)$ there is uniquely associated a dual irrep (with label $t=l+3 / 2$ ) of the $\mathrm{SU}(1,1)$ radial algebra [31]. Then, shift operators, $A^{\dagger}$ and $A[26]$, obtained by the factorization or SUSY QM approaches alluded to above, relate radial wave functions corresponding to different angular momenta. Specifically, the shift operators connect radial wave functions of the same $m_{t}$ but differing in $t$ by \pm 1 , i.e., radial wave functions associated with angular functions of angular momentum $l$ differing by \pm 1 . The algebraic structure (ladder operators) and shift operators in combination are what enable the evaluation of matrix elements of operators in the full three-dimensional space [20].

Let us now reexamine the choice of basis functions. Since we shall restrict attention to functions with radial-angular factorization, these would also commonly be termed orbitals. In actual calculations, the basis must be truncated to finite size (either the set of singleparticle basis functions may be truncated directly, or some more general limitation may be placed on the set of many-body antisymmetrized product states constructed from this basis [32]). Therefore, we must be concerned not only with mathematical completeness of the basis but also with how well matched the basis is to the physical problem at hand. That is, the basis functions should be chosen according to the following criteria (see Ref. [6]): (i) The functions should allow for a systematic approach to completeness as the single-particle basis set is expanded. (ii) The functions should provide for rapid convergence of the calculated energies or eigenfunctions to the true results, requiring only a small number of basis functions for an accurate description. (iii) It is also practically desirable that the functions have a simple analytic form which permits convenient evaluation of relevant operator matrix elements. (Ideally, the functions should also be orthogonal and easily normalized, to simplify the formulation of the many-body problem, but this is not essential.)

The harmonic oscillator eigenfunctions are of the form (for simplicity, we omit normal- 
ization factors and set the radial length parameter to unity) [3, 18]

$$
\Omega_{n l m}(\mathbf{r}) \propto r^{l+1} L_{n}^{l+1 / 2}\left(r^{2}\right) e^{-r^{2} / 2} Y_{l m}(\hat{\mathbf{r}}),
$$

where $L_{n}^{\alpha}(x)$ is the associated Laguerre polynomial of degree $n$ and order $\alpha$, i.e., for parameter $\alpha$ in the associated Laguerre equation [33], and $Y_{l m}(\hat{\mathbf{r}})$ is a spherical harmonic. These functions constitute a complete, orthonormal set on $\mathcal{L}^{2}\left(\mathbb{R}^{3}\right)$. However, while the Schrödinger equation solutions for a finite-range potential (or for an infinite-range potential of Coulomb $1 / r$ type) exhibit an exponential asymptotic decay at large $r\left(\propto e^{-\beta r}\right)$, the oscillator functions have Gaussian asymptotics $\left(\propto e^{-\alpha r^{2}}\right)$, as an artifact of the infinite (quadratic) binding potential of the harmonic oscillator problem. The oscillator functions are thus poorly matched to the physical solutions in the asymptotic region and may therefore violate criterion (ii) above for a suitable basis, i.e., rapid convergence. In nuclear physics, for instance, while structure involving only deeply-bound orbitals might be adequately represented using basis functions with Gaussian asymptotics, weakly-bound orbitals with distinctly exponential asymptotics influence many aspects of nuclear structure, especially in light or neutron-rich nuclei (see, e.g., Fig. 4 of Ref. [34] and Fig. 4 of Ref. [35]).

The Coulomb wave functions, as solutions to a problem with finite binding, display the appropriate exponential asymptotics. The bound-state (or negative-energy) Coulomb functions [3, 6, 33] are of the form [36]

$$
W_{n l m}(\mathbf{r}) \propto\left(\frac{2 r}{n+l+1}\right)^{l} L_{n}^{2 l+1}\left(\frac{2 r}{n+l+1}\right) e^{-r /(n+l+1)} Y_{l m}(\hat{\mathbf{r}}) .
$$

The full set of solutions to the Schrödinger equation with the Coulomb potential, including both these bound-state solutions and the continuum (or positive-energy) solutions, constitutes a complete set on $\mathcal{L}^{2}\left(\mathbb{R}^{3}\right)$. However, the bound-state solutions by themselves do not. This is not merely a formal deficiency: attempting to use them as an expansion basis is well-known to result in convergence to erroneous energies and wave functions [1]. It is therefore necessary to supplement the bound-state solutions with the continuum functions. The inconvenience of including continuum functions limits the practical applicability of the Coulomb basis: both in terms of arranging a systematic approach to completeness with increasing basis size [criterion (i)] and due to the complicated nature of the continuum wave functions [criterion (iii)] [3, 6].

One may instead seek a discrete complete set, retaining the same exponential asymptotics as the Coulomb wave functions, by modifying the Schrödinger equation into a Sturm- 
Liouville problem associated with the Coulomb potential (as detailed in Sec. II), thereby obtaining the Coulomb-Sturmian functions [3, 37 40]. These have been applied as expansion bases in atomic and molecular theory [37, 38, 41,43], nuclear scattering [44, 45], and other few-body problems [46]. The Coulomb-Sturmian functions are of the form

$$
\Psi_{n l m}(\mathbf{r}) \propto(2 r)^{l} L_{n}^{2 l+1}(2 r) e^{-r} Y_{l m}(\hat{\mathbf{r}})
$$

that is, of the same functional form as the Coulomb eigenproblem solutions (2) but independently rescaled in the radial coordinate so that $r /(n+l+1) \rightarrow r$. These radial functions constitute a discrete set, complete on the Sobolev space $\mathcal{W}_{2}^{(1)}\left(\mathbb{R}^{3}\right)[$ i.e., the space of all functions in $\mathcal{L}^{2}\left(\mathbb{R}^{3}\right)$, the generalized second derivatives of which are also in $\left.\mathcal{L}^{2}\left(\mathbb{R}^{3}\right)\right]$ ] , which implies completeness on $\mathcal{L}^{2}\left(\mathbb{R}^{3}\right)$ [47]. However, these functions are orthogonal with respect to the Coulomb potential $1 / r$ as weighting function, rather than the usual Euclidean metric.

We therefore finally turn to the Laguerre functions [48, 49] which are of the form

$$
\Lambda_{n l m}(\mathbf{r}) \propto(2 r)^{l} L_{n}^{2 l+2}(2 r) e^{-r} Y_{l m}(\hat{\mathbf{r}}) .
$$

They are identical in form to the Coulomb-Sturmian functions (3), differing only in the parameter $\alpha$ of the associated Laguerre polynomial: odd $\alpha=2 l+1$ for the CoulombSturmian functions vs. even $\alpha=2 l+2$ for the Laguerre functions [50]. The Laguerre functions satisfy all the posited criteria: consitituting a discrete set, complete on $\mathcal{L}^{2}\left(\mathbb{R}^{3}\right)$, and orthogonal with respect to the usual Euclidean metric, but with the physically-appropriate exponential asymptotics.

We demonstrate that an algebraic framework, analogous to that obtained for the usual central force eigenfunctions, can likewise be constructed for the Laguerre functions. These results permit analytic expressions to be obtained for matrix elements. The Laguerre functions are first introduced (Sec. II ). After a brief review of the $\mathrm{SO}(3)$ and $\mathrm{SU}(1,1)$ Lie algebras and the structure of their irreps (Sec. III A), we develop the $\mathrm{SU}(1,1)$ algebra of the Laguerre radial functions (Sec. IIIB), and we use this algebra to obtain analytic expressions for the action of several basic operators (involving powers of the radial coordinate $r$ and radial derivative operator $d / d r$ ) on the basis of Laguerre radial functions - or, equivalently, analytic expressions for matrix elements of these operators (Sec. III C). The results thus obtained apply to functions within a single $\mathrm{SU}(1,1)$ irrep, i.e., to the set of radial functions associated with the same angular momentum. We then extend the ideas of factorization (or SUSY 
QM) to a quantum number dependent formulation (Sec. IV A), to construct shift operators relating Laguerre radial functions in different irreps (Sec. IV B), and thus to obtain analytic expressions for the matrix elements of radial operators between different irreps (Sec. IV C). Finally, returning to the full Laguerre functions in three dimensions, we demonstrate how the results for radial matrix elements and shift operators are combined to yield reduced matrix elements of spherical tensor operators, taking the Laplacian and gradient operators for illustration (Sec. V).

\section{LAGUERRE FUNCTIONS}

The Coulomb functions (2) are the solutions to the Schrödinger equation

$$
\left(-\nabla^{2}-Z_{\frac{r}{r}}^{2}\right) W(\mathbf{r})=2 E_{n l} W(\mathbf{r}), \quad E_{n l}=-\frac{Z^{2}}{2(n+l+1)^{2}} .
$$

As outlined above, these do not form a complete set on $\mathcal{L}^{2}\left(\mathbb{R}^{3}\right)$ without including the continuum [1, 41], making them an impractical choice of basis.

There is, however, a set of functions, closely related to the Coulomb functions, called the Coulomb-Sturmian functions (3), which combine the discrete character of the harmonic oscillator functions with the desired large $r$ behavior. These functions are related to the Coulomb functions by making the substitution

$$
\frac{n+l+1}{Z} \rightarrow b,
$$

which recasts the differential equation (5) into the form of a Sturm-Liouville equation

$$
\left(-\nabla^{2}-\frac{2}{b} \frac{\beta_{n l}}{r}\right) \Psi(\mathbf{r})=-\frac{1}{b^{2}} \Psi(\mathbf{r}), \quad \beta_{n l}=n+l+1,
$$

and yields the Coulomb-Sturmian solutions

$$
\Psi_{n l m}(\mathbf{r})=\left(\frac{(2 / b)^{3} n !}{2(n+l+1)(n+2 l+1) !}\right)^{\frac{1}{2}}\left(\frac{2 r}{b}\right)^{l} L_{n}^{2 l+1}(2 r / b) e^{-r / b} Y_{l m}(\hat{r}) .
$$

Observe that, while in the Schrödinger equation eigenproblem (5) one varies the energy $E_{n l}$ to satisfy the boundary conditions, in the Sturm-Liouville eigenproblem (7) one now holds the energy constant and varies the coefficient $\beta_{n l}$, which governs the strength or depth of the potential, to satisfy the boundary conditions [38]. From the basic theory of the SturmLiouville equation [51], it follows that the solutions $\Psi_{n l m}$ are orthogonal with respect to the 
Coulomb potential $1 / r$ as weighting function [38]. These functions $\Psi_{n l m}$ are also complete in the Sobolev space $\mathcal{W}_{2}^{(1)}\left(\mathbb{R}^{3}\right)$ [3].

However, for calculations in physics it is useful to work with functions that are orthonormal with respect to the usual integration metric $d^{3} r$ and complete on $\mathcal{L}^{2}\left(\mathbb{R}^{3}\right)$. To obtain functions with this property, the integration weight and norm are absorbed into the function by multiplying $\Psi_{n l m}$ by $[b(n+l+1) / r]^{1 / 2}$, and the shift $l \rightarrow l+1 / 2$ is introduced [3]. The resulting functions are the Laguerre functions,

$$
\Lambda_{n l m}(\mathbf{r})=\left(\frac{2}{b}\right)^{\frac{3}{2}}\left(\frac{n !}{(n+2 l+2) !}\right)^{\frac{1}{2}}\left(\frac{2 r}{b}\right)^{l} L_{n}^{2 l+2}(2 r / b) e^{-r / b} Y_{l m}(\hat{r}),
$$

which satisfy the radial differential equation

$$
\left[-\frac{\partial^{2}}{\partial r^{2}}-\frac{3}{r} \frac{\partial}{\partial r}+\frac{l(l+2)}{r^{2}}-\frac{2}{b} \frac{\alpha_{n l}}{r}\right] \Lambda_{n l m}(\mathbf{r})=-\frac{1}{b^{2}} \Lambda_{n l m}(\mathbf{r}), \quad \alpha_{n l}=n+l+\frac{3}{2} .
$$

The Laguerre functions $\Lambda_{n l m}(\mathbf{r})$ form a complete, discrete basis for $L^{2}\left(\mathbb{R}^{3}\right)$ [47, have the correct asymptotic behavior and are orthogonal with respect to the $\mathbb{R}^{3}$ metric. That is,

$$
\int d^{3} r \Lambda_{n^{\prime} l^{\prime} m^{\prime}}(\mathbf{r}) \Lambda_{n l m}(\mathbf{r})=\delta_{n, n^{\prime}} \delta_{l, l^{\prime}} \delta_{m, m^{\prime}}
$$

It is now the matrix elements calculated in this Laguerre basis that are of interest. Certain matrix elements can be calculated via direct integration [8, 10]. However, in this paper we use the factorability of the Hilbert space to develop a simpler and more elegant algebraic method for calculating matrix elements.

The Laguerre function $\Lambda_{n l m}(\mathbf{r})$ can be factorized into radial and angular functions as

$$
\Lambda_{n l m}(\mathbf{r})=r^{-1} S_{n l}(r) Y_{l m}(\hat{\mathbf{r}}) .
$$

The integration weight has been absorbed into the radial function $S_{n l}(r)$, given by

$$
S_{n l}(r)=\left(\frac{2}{b}\right)^{\frac{1}{2}}\left(\frac{n !}{(n+2 l+2) !}\right)^{\frac{1}{2}}\left(\frac{2 r}{b}\right)^{l+1} L_{n}^{2 l+2}(2 r / b) e^{-r / b},
$$

which satisfies the orthogonality condition

$$
\int_{0}^{\infty} d r S_{n^{\prime} l}(r) S_{n l}(r)=\delta_{n, n^{\prime}}
$$

The set of functions $\left\{S_{n l} \mid n=0,1,2, \ldots\right\}$ forms a complete and orthogonal set on $L^{2}\left(\mathbb{R}^{+}\right)$, for any $l=0,1,2, \ldots$, independent of $b[39]$, so we make the simplifying substitution $b \rightarrow 1$ in the following discussion. The resulting functions,

$$
S_{n l}(r)=\left(\frac{2 n !}{(n+2 l+2) !}\right)^{\frac{1}{2}}(2 r)^{l+1} L_{n}^{2 l+2}(2 r) e^{-r}
$$


satisfy the differential equation

$$
\left[-\frac{d^{2}}{d r^{2}}-\frac{1}{r} \frac{d}{d r}+\frac{(l+1)^{2}}{r^{2}}-\frac{2 \alpha_{n l}}{r}\right] S_{n l}(r)=-1 S_{n l}(r),
$$

with $\alpha_{n l}$ as defined in (10). Although (16) is not explicitly written in the form of a SturmLiouville equation (see footnote [51]), it nonetheless may readily be put in this form by left multiplying by $r$. For convenience of notation, we will sometimes denote $\Lambda_{n l m}$ as $|n l m\rangle, S_{n l}$ as $|n l\rangle$ and $Y_{l m}$ as $|l m\rangle$.

\section{A. Operator map to the radial basis}

Although we want the matrix elements in the $\Lambda_{n l m}$ basis, the actual calculations will be carried out more simply using the functions

$$
|n l\rangle|l m\rangle,
$$

since they are orthogonal with respect to the measure $d r d \Omega$ rather than the measure $r^{2} d r d \Omega$. We start with the matrix element of a component of some irreducible, rank $a$ tensor operator

$$
\mathcal{O}_{a \alpha}=R \Theta_{a \alpha},
$$

where $R$ is a scalar radial operator and $\Theta_{a}$ is a rank $a$ tensor with no radial dependence. Although we are only considering operators that can be factored into radial and angular parts, many operators of interest can be rewritten as sums of such separable operators using various factorization lemmas, and so this method can also be used to calculate matrix

elements of these operators. As an example, matrix elements of the gradient operator $\nabla_{1}$ are calculated in Sec. VC.

The matrix element of the operator $\mathcal{O}_{a \alpha}$ separates into radial and angular factors as

$$
\begin{aligned}
\left\langle n^{\prime} l^{\prime} m^{\prime}\left|\mathcal{O}_{a \alpha}\right| n l m\right\rangle= & \int d^{3} r \Lambda_{n l m}(\mathbf{r}) \mathcal{O}_{a \alpha} \Lambda_{n l m}(\mathbf{r}) \\
& =\left[\int d r r^{2} r^{-1} S_{n^{\prime} l^{\prime}}(r) R(r) r^{-1} S_{n l}(r)\right]\left[\int d \Omega Y_{l^{\prime} m^{\prime}}(\hat{r}) \Theta_{a \alpha} Y_{l m}(\hat{r})\right]
\end{aligned}
$$

That is,

$$
\left\langle n^{\prime} l^{\prime} m^{\prime}\left|\mathcal{O}_{a \alpha}\right| n l m\right\rangle=\left\langle n^{\prime} l^{\prime}|\gamma(R)| n l\right\rangle\left\langle l^{\prime} m^{\prime}\left|\Theta_{a \alpha}\right| l m\right\rangle,
$$

where

$$
\gamma(R)=r R r^{-1}
$$


If the operator is purely radial $\left(\Theta_{a \alpha} \rightarrow \mathbf{1}_{00}\right)$, then we have that

$$
\left\langle n^{\prime} l^{\prime} m^{\prime}|R| n l m\right\rangle=\left\langle n^{\prime} l|\gamma(R)| n l\right\rangle
$$

The similarity transformation given by (21) will allow us to calculate matrix elements in the $\Lambda_{n l m}$ basis by factoring the matrix element into linear combinations of products of a matrix element in the $S_{n l}$ basis and a matrix element in the well known angular momentum basis, as shown (for the case of positive weights) in Sec. V.

\section{ALGEBRAIC STRUCTURE OF LAGUERRE BASIS}

The factorization 12 of the $\Lambda_{n l m}$ basis for $\mathcal{L}\left(\mathbb{R}^{3}\right)$ has the underlying symmetry $\mathrm{SU}(1,1) \times \mathrm{SO}(3)$, where $\mathrm{SU}(1,1)$ is the symmetry of the radial functions and $\mathrm{SO}(3)$ in the symmetry of $Y_{l m}$ angular functions. It is well known that the $\mathrm{SO}(3)$ symmetry can be used to calculate matrix elements of operators on the $Y_{l m}$ basis. In this section we discuss how $\mathrm{SU}(1,1)$ symmetry can be used to calculate the matrix elements on the $S_{n l}$ basis.

\section{A. A brief review of the $\mathrm{SO}(3)$ and $\mathrm{SU}(1,1)$ Lie algebras}

The Lie algebra $\mathrm{SU}(1,1)$ has a very similar structure to that of the Lie algebra $\mathrm{SO}(3)$, so we begin by summarizing the properties of the more familiar $\mathrm{SO}(3)$ algebra for comparison with the $\mathrm{SU}(1,1)$ algebra.

The group $\mathrm{SO}(3)$ is the compact Lie group of rotations in three dimensions. Its algebra is generated by the operators $L_{3}$ and $L_{ \pm}$, which satisfy the commutation relations

$$
\left[L_{3}, L_{ \pm}\right]= \pm L_{ \pm}
$$

and

$$
\left[L_{+}, L_{-}\right]=2 L_{3}
$$

where $L_{+}^{\dagger}=L_{-}$. The eigenfunctions of $L_{3}$ are the spherical harmonics $|l m\rangle$, where

$$
L_{3}|l m\rangle=m|l m\rangle
$$

The laddering operators $L_{ \pm}$have the well known action on the spherical harmonics

$$
L_{ \pm}|l m\rangle=\sqrt{(l \mp m)(l \pm m+1)}|l, m \pm 1\rangle .
$$




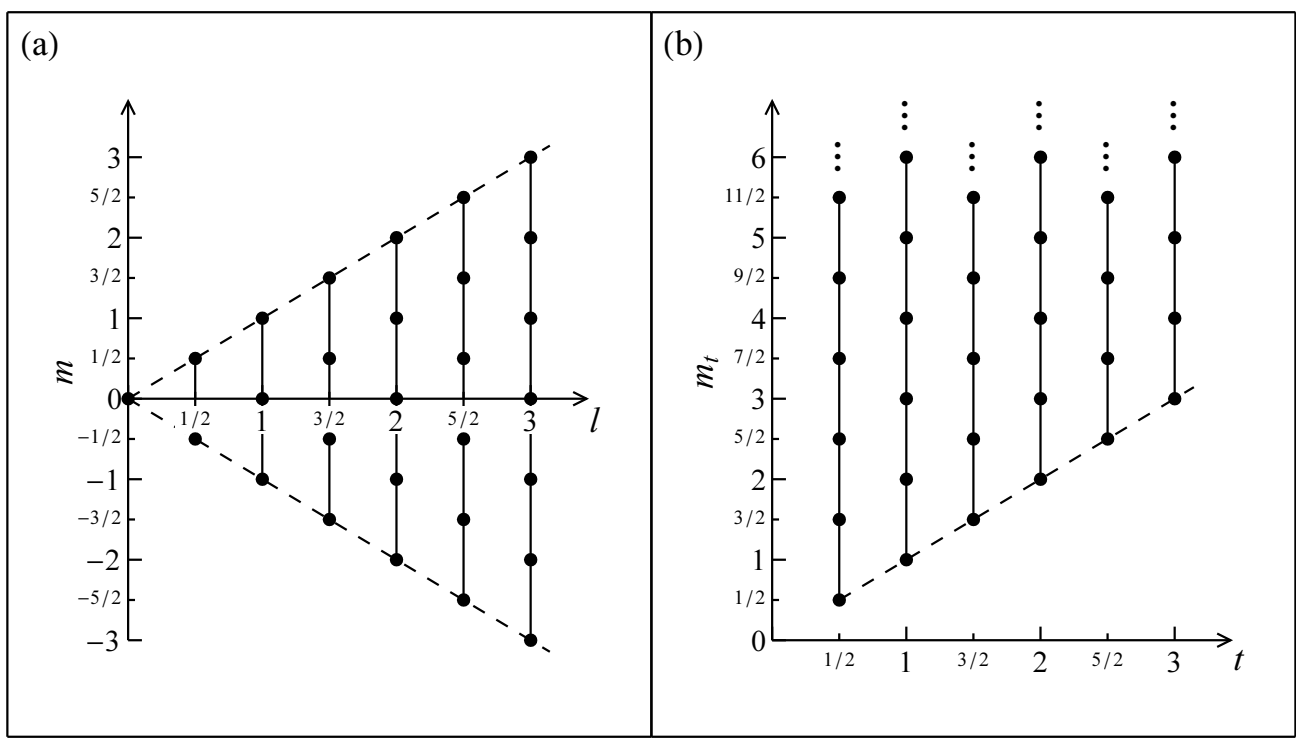

FIG. 2: Weights contained within (a) $\mathrm{SO}(3)$ and (b) $\mathrm{SU}(1,1)$ irreps of the types considered in the present work (see text). Weights ( $m$ or $m_{t}$, respectively, for these two algebras) within an irrep (labeled by extremal weight $l$ or $t$, respectively) are connected by solid lines. The bounding weights, as functions of $l$ or $t$, are indicated by dashed lines.

Because $\mathrm{SO}(3)$ is a compact group, the weight $m$ is bounded above and below. The bound is determined by the $\mathrm{SO}(3)$ Casimir operator

$$
C_{S O(3)}=L_{3}^{2}+\frac{1}{2}\left(L_{+} L_{-}+L_{-} L_{+}\right)
$$

which acts on the eigenfunctions as

$$
C_{S O(3)}|l m\rangle=l(l+1)|l m\rangle .
$$

The bounds above and below on $m$ are given by $\pm l$, that is

$$
m=l, l-1, \ldots,-l,
$$

as shown in Fig. 2(a).

Similarly, the generators of $\mathrm{SU}(1,1)$ are $T_{3}$ and $T_{ \pm}$, which satisfy the commutation relations:

$$
\left[T_{3}, T_{ \pm}\right]= \pm T_{ \pm}
$$

and

$$
\left[T_{+}, T_{-}\right]=-2 T_{3}
$$


where $T_{+}^{\dagger}=T_{-}$. The actions of the generators on the eigenfunctions $\left|t m_{t}\right\rangle$ of $T_{3}$ are given by

$$
T_{3}\left|t m_{t}\right\rangle=m_{t}\left|t m_{t}\right\rangle
$$

and

$$
T_{ \pm}\left|t m_{t}\right\rangle=\sqrt{\left(t \pm m_{t}\right)\left(t \mp m_{t}-1\right)}\left|t, m_{t} \pm 1\right\rangle .
$$

The commutator in (31) differs from the commutator in 24 by a negative sign. The group $\mathrm{SU}(1,1)$ is a non-compact group, and the weight $m_{t}$ is either bounded above or bounded below but takes on infinitely many values. As with $\mathrm{SO}(3)$, the bound on $m_{t}$ is determined by the Casmir operator

$$
C_{S U(1,1)}=T_{3}^{2}-\frac{1}{2}\left(T_{+} T_{-}+T_{-} T_{+}\right),
$$

which acts on the eigenfunction as

$$
C_{S U(1,1)}\left|t m_{t}\right\rangle=t(t-1)\left|t m_{t}\right\rangle .
$$

If $t$ is an integer or half integer, then the irrep is discrete. If $t>0$ then the irrep is bounded below by $t$, and if $t<0$ then the irrep is bounded above by $t$. In other words,

$$
m_{t}=\left\{\begin{array}{ll}
t, t+1, \ldots & t>0 \\
-t,-t-1, \ldots & t<0
\end{array},\right.
$$

as shown in Fig. 2(b) for $t>0$.

\section{B. Representation of $\mathrm{SU}(1,1)$ on the Laguerre radial basis}

In this section, we construct a representation of the Lie algebra of the radial group, $\mathrm{SU}(1,1)$, using the $S_{n l}(r)$ radial functions as the basis of the vector space. Recall from (16) that the $S_{n l}$ basis is generated by varying $\alpha_{n l}$. We rearrange (16) to rewrite it as an eigenvalue problem for $\alpha_{n l}$ :

$$
\left(-r \frac{d^{2}}{d r^{2}}-\frac{d}{d r}+\frac{(l+1)^{2}}{r}+r\right) S_{n l}(r)=2 \alpha_{n l} S_{n l}(r)
$$

Following a similar approach to the method used in [21, 26] to construct representations of $\mathrm{SU}(1,1)$ for the Coulomb functions, we define

$$
T_{3}=-\frac{1}{2}\left(r \frac{d^{2}}{d r^{2}}+\frac{d}{d r}-\frac{(l+1)^{2}}{r}-r\right)
$$


and

$$
T_{ \pm}=-\frac{1}{2}\left(r \frac{d^{2}}{d r^{2}}+\frac{d}{d r}-\frac{(l+1)^{2}}{r}+r \mp 2 r \frac{d}{d r} \mp 1\right)
$$

which satisfy the commutation relations in 30 and (31) and the relation $T_{+}^{\dagger}=T_{-}$. The Casimir invariant is given by

$$
C_{\mathrm{SU}(1,1)}=T_{3}^{2}-\frac{1}{2}\left(T_{+} T_{-}+T_{-} T_{+}\right)=\left(l+\frac{3}{2}\right)\left(l+\frac{1}{2}\right) .
$$

Thus the representation is discrete, with $t=l+3 / 2$ and weights $m_{t}=n+l+3 / 2(n=$ $0,1, \ldots$.$) . Note that m_{t}=\alpha_{n l}$, with $\alpha_{n l}$ as defined in (10). From (32) and (33), the actions of the generators are expressed in terms of the labels $n$ and $l$ as

$$
\begin{gathered}
T_{3} S_{n l}=\left(n+l+\frac{3}{2}\right) S_{n l} \\
T_{+} S_{n l}=\sqrt{(n+1)(n+2 l+3)} S_{n+1, l} \\
T_{-} S_{n l}=\sqrt{n(n+2 l+2)} S_{n-1, l} .
\end{gathered}
$$

\section{Deriving the action of radial operators using the $S U(1,1)$ algebra}

We can use the $\mathrm{SU}(1,1)$ algebra to easily determine the actions of $r, 1 / r$ and $d / d r$ on the functions $S_{n l}(r)$ following a method similar to that used by Rowe in [23] to calculate the matrix elements for the generalized harmonic oscillator functions. We begin with the elements of the algebra

$$
r=\frac{1}{2}\left(2 T_{3}-T_{+}-T_{-}\right)
$$

and

$$
r \frac{d}{d r}=\frac{1}{2}\left(T_{+}-T_{-}-1\right) .
$$

Applying the operators in (44) and (45) to $S_{n l}$, we immediately have that

$$
\begin{aligned}
r S_{n l}(r)=\left(n+l+\frac{3}{2}\right) S_{n l}(r)-\frac{1}{2} \sqrt{n(n+2 l+2)} & S_{n-1, l}(r) \\
& -\frac{1}{2} \sqrt{(n+1)(n+2 l+3)} S_{n+1,1}(r)
\end{aligned}
$$

and

$$
\begin{aligned}
r \frac{d}{d r} S_{n l}(r)=\frac{1}{2} \sqrt{(n+1)(n+2 l+3)} S_{n+1, l}( & \\
& -\frac{1}{2} \sqrt{n(n+2 l+2)} S_{n-1, l}(r)-\frac{1}{2} S_{n l}(r) .
\end{aligned}
$$


We can obtain the action of the operator $1 / r$, which is not an element of the $\mathrm{SU}(1,1)$ algebra, using a recurrence relationship that we deduce using the orthogonality of the $S_{n l}(r)$ functions. Starting with (46), we divide through by $r$, multiply through by $S_{m l}(r)$ and integrate to obtain

$$
\delta_{n, m}=\left(n+l+\frac{3}{2}\right) f_{n m}-\frac{1}{2} \sqrt{n(n+2 l+2)} f_{n-1, m}-\frac{1}{2} \sqrt{(n+1)(n+2 l+3)} f_{n+1, m},
$$

where

$$
f_{n m}=\int_{0}^{\infty} d r S_{m l}(r) \frac{1}{r} S_{n l}(r) .
$$

Starting with $f_{00}=1 /(l+1)$, obtained via direct integration, the recurrence relationship 48) can be solved to find that

$$
f_{n m}=\frac{1}{l+1} \sqrt{\frac{n !(m+2 l+2) !}{m !(n+2 l+2) !}}, \quad n \geq m .
$$

A similar result, obtained by simply interchanging $m$ and $n$, holds for $n<m$. From these results we have that

$$
\frac{1}{r} S_{n l}(r)=\frac{1}{l+1} \sum_{i \geq n+1} \sqrt{\frac{i !(n+2 l+2) !}{n !(i+2 l+2) !}} S_{i l}(r)+\frac{1}{l+1} \sum_{i \leq n} \sqrt{\frac{n !(i+2 l+2) !}{i !(n+2 l+2) !}} S_{i l}(r) .
$$

Finally, the action of the radial derivative can be determined by noting that $d / d r=$ $r^{-1}(r d / d r)$. Combining (47) and (51),

$$
\frac{d}{d r} S_{n l}(r)=\sum_{i \geq n+1} \sqrt{\frac{i !(n+2 l+2) !}{n !(i+2 l+2) !}} S_{i l}(r)-\sum_{i \leq n-1} \sqrt{\frac{n !(i+2 l+2) !}{i !(n+2 l+2) !}} S_{i l}(r) .
$$

The actions of other operators, built from these basic operators, can be calculated by combining (46), (51) and (52).

Once we have the action of a radial operator $R(r)$ on $S_{n l}$, we can extract the matrix element $\left\langle n^{\prime} l|R(r)| n l\right\rangle$ by inspection as the coefficient of $S_{n^{\prime} l}$ in the sum, using the orthogonality of the radial functions $S_{n, l}$. For example, if $n^{\prime} \geq n+1$, then

$$
\begin{aligned}
\left\langle n^{\prime} l\left|\frac{d}{d r}\right| n l\right\rangle & =\sum_{i \geq n+1} \sqrt{\frac{i !(n+2 l+2) !}{n !(i+2 l+2) !}} \delta_{n^{\prime} i}-\sum_{i \leq n-1} \sqrt{\frac{n !(i+2 l+2) !}{i !(n+2 l+2) !}} \delta_{n^{\prime} i} \\
& =\sqrt{\frac{n^{\prime} !(n+2 l+2) !}{n !\left(n^{\prime}+2 l+2\right) !}}
\end{aligned}
$$

Expression for the matrix elements for $r, r^{2}, 1 / r, 1 / r^{2}, r d / d r, d / d r$ and $d^{2} / d r^{2}$ are given in Appendix A (Table I). 


\section{SHIFT OPERATORS}

In the previous section, we derived expressions for matrix elements of operators between Laguerre radial functions within the same $\mathrm{SU}(1,1)$ irrep, i.e., matrix elements between the three-dimensional basis functions with the same angular quantum number $l$. However, many operators of interest in physics are not strictly radial, scalar operators (e.g., $\mathbf{r}$ and $\boldsymbol{\nabla}$ are vector operators or, equivalently, spherical tensors of rank 1) and may therefore have nonvanishing matrix elements between states with different $l$. If we consider a spherical tensor operator $\mathcal{O}_{a \alpha}$ which factorizes into radial and angular parts, as discussed in Sec. IIA, then recall from (20) that the problem reduces to evaluating the radial matrix element $\left\langle n^{\prime} l^{\prime}|\gamma(R)| n l\right\rangle$, with $l^{\prime} \neq l$, that is, connecting Laguerre radial functions lying in different $\mathrm{SU}(1,1)$ irreps.

The matrix elements of radial operators between radial functions in different irreps $\left(l^{\prime} \neq l\right)$ can be written in terms of the matrix elements we have already calculated, i.e., between members of the same irrep $\left(l^{\prime}=l\right)$, provided we can obtain analytic relations allowing us to expand the members of one of the irreps (namely, $l^{\prime}$ ) as linear combinations of those in the other irrep (namely, $l$ ). This relation may be obtained through shift operators, $A^{\dagger}$ and $A$, introduced in Sec. I.

To see how we may obtain such shift operators, recall from (16) that the Laguerre functions are eigenfunctions of a differential operator. In particular, the operator

$$
H_{n l}=-\frac{d^{2}}{d r^{2}}-\frac{1}{r} \frac{d}{d r}+\frac{(l+1)^{2}}{r^{2}}-\frac{2 \alpha_{n l}}{r}
$$

may be taken as a Hamiltonian operator defining the Laguerre functions (of a single irrep l) as its eigenfunctions, except that this Hamiltonian involves an $n$-dependent coefficient $\alpha_{n l}$ and is thus actually a quantum number dependent Hamiltonian (the eigenfunctions of interest are also degenerate, with eigenvalue -1 ).

The problem of relating Laguerre functions from different irreps (of different $l$ ) thus amounts to the problem of relating the eigenfunctions of a sequence of Hamiltonians, which are defined through discrete variation of a parameter $l$. This is the problem addressed by the factorization method of Infeld and Hull [29] or, equivalently, for present purposes, the factorization method used in supersymmetric quantum mechanics (SUSY QM) [30] for shape invariant potentials [52]. 


\section{A. Factorization with quantum number dependence}

The essential idea of factorization is that, if a Hamiltonian can be written in a factorized form $H=A^{\dagger} A$, this immediately gives rise to a partner Hamiltonian $H^{\prime}=A A^{\dagger}$, with (at least some) eigenvalues which are degenerate to those of $H$, and with eigenfunctions which are related to those of $H$ - in fact, related by the action of the operators $A^{\dagger}$ and $A$. Factorization is a particularly powerful technique in problems where we have an extended (perhaps infinite) sequence or "tower" of related Hamiltonians $H_{m}=A_{m}^{\dagger} A_{m}(m=1,2$, $\ldots)$, where $m$ is a discrete parameter in the Hamiltonian. Then we likewise have extended sets of degenerate states $\psi_{n m}(n=0,1, \ldots$ and $m=1,2, \ldots)$, related to each other by the action of the operators $A_{m}^{\dagger}$ and $A_{m}$. SUSY QM provides a general formalism for finding such a factorization, through the superpotential [30]. However, for many familiar exactlysolvable Schrödinger equation problems, the factorizations have been cataloged by Infeld and Hull [29, and the problem of finding a factorization reduces to that of transforming the problem into one of their standard forms.

It may be helpful to note both the analogy to the familiar factorization of the onedimensional harmonic oscillator Hamiltonian, $H=a^{\dagger} a+1 / 2$, explored by Dirac [53], as well as the differences from this factorization. In the oscillator problem, the operators $a^{\dagger}$ and $a$ are simply laddering operators, between eigenfunctions of the same Hamiltonian, serving to change the number of nodes. However, the operators $A^{\dagger}$ and $A$ in the more general factorization are shift operators, between eigenfunctions of different Hamiltonians, though in fact their action also changes the number of nodes.

While factorization is a powerful technique for solving eigenproblems, we are interested here primarily in the shift operation which it provides, towards our goal of relating Laguerre radial functions in different irreps. We therefore summarize the necessary ideas behind the shift operation, then show how the relevant relations can readily be extended to accomodate quantum number dependent Hamiltonians, such as encountered for the Laguerre functions in (54). We refer the reader to the more extensive discussions in Refs. [29, 30, 54] for a more comprehensive discussion of the families of potentials which lead to factorizable Hamiltonians, the solution process, and the nature of the eigenspectra which result. Our notational conventions for factorization follow those of, e.g., Refs. [23, 30, in the use of $A^{\dagger}$ and $A$ for the shift operators. 
To start with this simplest case, of two partner Hamiltonians, suppose the Hamiltonian for some one-dimensional eigenproblem can be written as

$$
H_{1}(r)=A_{1}^{\dagger}(r) A_{1}(r)+K_{1}
$$

where, in addition to the factorized portion of the Hamiltonian, it is convenient to permit a constant term $K_{1}$. (Since the primary concern in the present work is with radial wave functions on $\mathbb{R}^{+}$, we have chosen to denote the independent variable as $r$ rather than as $x$, but the discussion here is relevant without modification to one-dimensional problems on $\mathbb{R}$.) Then the eigenspectrum of $H_{1}$ is related in a simple way to that of the partner Hamiltonian

$$
H_{2}(r)=A_{1}(r) A_{1}^{\dagger}(r)+K_{1}
$$

Specifically, suppose $H_{1}$ has eigenfunctions $\psi_{n 1}(n=0,1, \ldots)$, with associated eigenvalues $E_{n 1}$, and $H_{2}$ has eigenfunctions $\psi_{n 2}(n=0,1, \ldots)$, with associated eigenvalues $E_{n 2}$, that is,

$$
\begin{aligned}
& H_{1} \psi_{n 1}=E_{n 1} \psi_{n 1} \\
& H_{2} \psi_{n 2}=E_{n 2} \psi_{n 2} .
\end{aligned}
$$

Then, simply from the form of (55) and (56), one can readily verify (as we shall illustrate below in a more general context) that acting with $A_{1}$ on any eigenfunction $\psi_{n 1}$ of $H_{1}$ gives an eigenfunction of $H_{2}$, still with eigenvalue $E_{n 1}$. Similarly, acting with $A_{1}^{\dagger}$ on any eigenfunction $\psi_{n 2}$ of $H_{2}$ gives an eigenfunction of $H_{1}$, still with eigenvalue $E_{n 2}$. Thus, eigenfunctions of $H_{1}$ and $H_{2}$ must occur as degenerate partners. The exception arises if $A_{1}$ (or $A_{1}^{\dagger}$ ) annihilates the wave function on which it acts, that is, if it yields the null function instead of a bona fide eigenfunction of the partner Hamiltonian. We shall restrict our attention to the typical case (see Refs. [29, 30, 54]) in which $A_{1} \psi_{01}=0$. That is, the ground state eigenfunction of $H_{1}$ has no partner. Then the remaining eigenfunctions and eigenvalues follow the correspondence $($ for $n=1,2, \ldots)$

$$
\begin{aligned}
\psi_{n 1}(r) & \propto A_{1}^{\dagger}(r) \psi_{n-1,2}(r) \\
\psi_{n-1,2}(r) & \propto A_{1}(r) \psi_{n 1}(r) \\
E_{n-1,2} & =E_{n 1} .
\end{aligned}
$$

The factorization process may, however, continue. Suppose $H_{2}$, in turn, can be refactored in some way, distinct from (56), as

$$
H_{2}(r)=A_{2}^{\dagger}(r) A_{2}(r)+K_{2} .
$$


Then the same pattern can be followed, as above, to relate the eigenspectrum of $\mathrm{H}_{2}$ to that of a partner Hamiltonian

$$
H_{3}(r)=A_{2}(r) A_{2}^{\dagger}(r)+K_{2}
$$

and relations on the eigenfunctions and eigenvalues analogous to $(59)-(61)$ can be obtained.

More generally, we may consider an infinite "tower" of related Hamiltonians $H_{m}$, parametrized by a discrete index $m(m=1,2, \ldots)$, where successive Hamiltonians $H_{m}$ and $H_{m+1}$ have related factorizations

$$
\begin{gathered}
H_{m}(r)=A_{m}^{\dagger}(r) A_{m}(r)+K_{m} \\
H_{m+1}(r)=A_{m}(r) A_{m}^{\dagger}(r)+K_{m} .
\end{gathered}
$$

We now have a sequence of related eigenproblems

$$
H_{m}(r) \psi_{n m}(r)=E_{n m} \psi_{n m}(r)
$$

Their eigenfunctions and eigenvalues are related by

$$
\begin{aligned}
\psi_{n m}(r) & \propto A_{m}^{\dagger}(r) \psi_{n-1, m+1}(r) \\
\psi_{n-1, m+1}(r) & \propto A_{m}(r) \psi_{n m}(r) \\
E_{n-1, m+1} & =E_{n m},
\end{aligned}
$$

etc. The identification of $n$ quantum numbers $(n=0,1, \ldots)$ across the different eigenproblems here again rests on the further conventional condition that the ground state for each $H_{m}$ be annihilated by the $A$ operator, i.e., $A_{m} \psi_{0 m}=0$ or, equivalently, as may be verified, $E_{0 m}=K_{m}$.

Let us take a moment to interpret the energy and operator relations indicated in (67) 69, which are illustrated schematically in Fig. 3. From 67), it is seen that the operator $A_{m}^{\dagger}$ acts on the wave function $\psi_{n-1, m+1}$ to give the wave function $\psi_{n m}$. It thus raises the quantum number $n$, which counts the number of nodes in the wave function, and is therefore reminiscent of Dirac's $a^{\dagger}$ operator. However, the initial and final wave functions are eigenfunctions of different Hamiltonians, as the Hamiltonian index has simultaneously been lowered. Thus, $A_{m}^{\dagger}$ is not merely a raising operator, but rather a shift operator. Similarly, from (68), it is seen that $A_{m}$ is likewise a shift operator, but with the inverse action on the node number and Hamiltonian indices. Finally, from (69), the eigenvalues form 


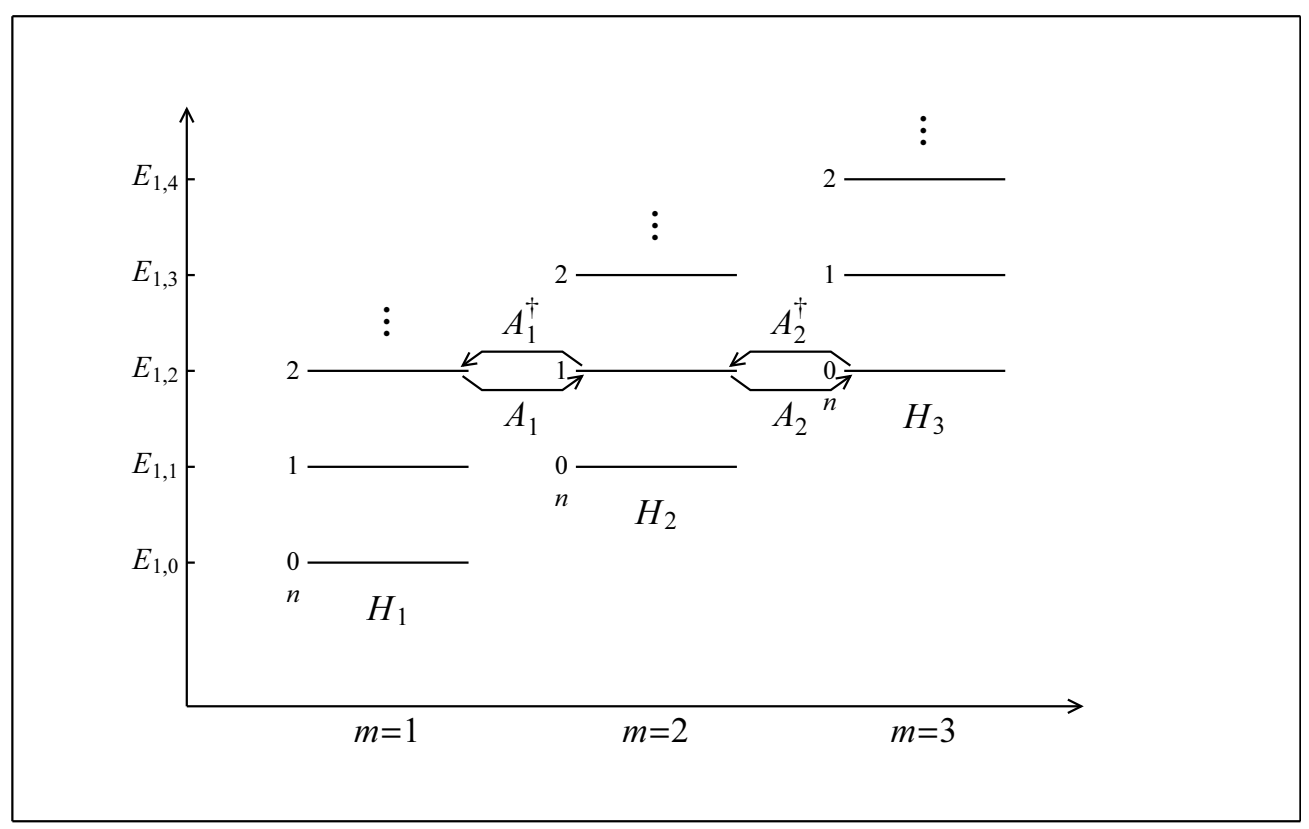

FIG. 3: Structure of the relations among eigenfunctions and eigenvalues for a sequence of factorized Hamiltonians $H_{m}$, defined in $67-69$.

degenerate multiplets across the different Hamiltonians, with $E_{n 0}=E_{n-1,1}=E_{n-2,2}=\ldots$. In fact, by repeated application of (67)-(69), the eigenfunctions and eigenvalues of any two Hamiltonians in the sequence can be related. Comparing the shift relations among eigenspectra arising from factorization (Fig. 3) with those anticipated for shift operators between $\mathrm{SU}(1,1)$ irreps (Fig. 1), we may already see the essential resemblance.

We may now generalize these relations to the case in which the Hamiltonians are $n$ dependent, that is, to eigenproblems of the form

$$
H_{n m}(r) \psi_{n m}(r)=E_{n m} \psi_{n m}(r)
$$

The factorizations (64) and 65) then generalize to

$$
\begin{aligned}
H_{n m}(r) & =A_{n m}^{\dagger}(r) A_{n m}(r)+K_{n m} \\
H_{n-1, m+1}(r) & =A_{n m}(r) A_{n m}^{\dagger}(r)+K_{n m},
\end{aligned}
$$

again with the assumption that the ground state eigenfunctions are annihilated, i.e., $A_{0 m}(r) \psi_{0 m}(r)=0$. We label the latter Hamiltonian with the index $n-1$, rather than $n$, in recognition of the anticipated correspondence of eigenfunctions across the Hamiltonians. 
We may now obtain $n$-dependent shift operator relations

$$
\begin{aligned}
\psi_{n m}(r) & \propto A_{n m}^{\dagger}(r) \psi_{n-1, m+1}(r) \\
\psi_{n-1, m+1}(r) & \propto A_{n m}(r) \psi_{n m}(r),
\end{aligned}
$$

while the eigenvalue relation $E_{n-1, m+1}=E_{n m}$ from $(69)$ still holds. These relations follow by first establishing that $A_{n m}^{\dagger}$ acts on the eigenfunction $\psi_{n-1, m+1}$ of $H_{n-1, m+1}$ to give an eigenfunction of $H_{n m}$, with the same eigenvalue $E_{n-1, m+1}$ :

$$
\begin{aligned}
H_{n m}\left[A_{n m}^{\dagger}(r) \psi_{n-1, m+1}(r)\right] & =\left[A_{n m}^{\dagger}(r) A_{n m}(r)+K_{n m}\right] A_{n m}^{\dagger}(r) \psi_{n-1, m+1}(r) \\
& =A_{n m}^{\dagger}(r)\left[A_{n m}(r) A_{n m}^{\dagger}(r)+K_{n m}\right] \psi_{n-1, m+1}(r) \\
& =A_{n m}^{\dagger}(r) H_{n-1, m+1} \psi_{n-1, m+1}(r) \\
& =E_{n-1, m+1}\left[A_{n m}^{\dagger}(r) \psi_{n-1, m+1}(r)\right],
\end{aligned}
$$

where we have successively applied the definition of $H_{n m}$ from (71), reassociated, recognized the definition of $H_{n-1, m+1}$ from $(72)$, and applied the eigenproblem definition from (70). It then may be verified, by a similar argument, that $A_{n m}$ acts on the eigenfunction $\psi_{n m}$ of $H_{n m}$ to give an eigenfunction of $H_{n-1, m+1}$, with the same eigenvalue $E_{n m}$, i.e.,

$$
H_{n-1, m+1}\left[A_{n m}(r) \psi_{n m}(r)\right]=E_{n m}\left[A_{n m}(r) \psi_{n m}(r)\right]
$$

To apply the shift operators in practice, it is desirable not only to know that a proportionality exists, as in (73) and (74), but also to know the constant of proportionality, so that we may construct normalized shift operators $\mathcal{A}_{n m}^{\dagger}$ and $\mathcal{A}_{n m}$. We require that these operators, when acting on normalized eigenfunctions, then yield normalized eigenfunctions, so that the proportionalities $(73)$ and $(74)$ become equalities

$$
\begin{aligned}
\psi_{n m}(r) & =\mathcal{A}_{n m}^{\dagger}(r) \psi_{n-1, m+1}(r) \\
\psi_{n-1, m+1}(r) & =\mathcal{A}_{n m}(r) \psi_{n m}(r) . .
\end{aligned}
$$

The constants of proportionality in (73) and (74) follow uniquely, within phase, from the assumed adjoint relationship between the operators $A_{n m}^{\dagger}$ and $A_{n m}$ and from the requirement that the wave functions $\psi_{n m}$ be normalized. In particular, the norm of $A_{n m}^{\dagger} \psi_{n-1, m+1}$ is readily evaluated, since (we switch to bracket notation to facilitate representing the inner 
product)

$$
\begin{aligned}
\left|A_{n m}^{\dagger} \psi_{n-1, m+1}\right|^{2} & =\left\langle\psi_{n-1, m+1}\left|A_{n m} A_{n m}^{\dagger}\right| \psi_{n-1, m+1}\right\rangle \\
& =\left\langle\psi_{n-1, m+1}\left|\left(H_{n-1, m+1}-K_{n m}\right)\right| \psi_{n-1, m+1}\right\rangle \\
& =E_{n-1, m+1}-K_{n m},
\end{aligned}
$$

where we have recognized the relationship between $A_{n m} A_{n m}^{\dagger}$ and $H_{n-1, m+1}$ by $(72$ and then applied the eigenproblem definition from (70). The result may simply be rewritten as $\left|A_{n m}^{\dagger} \psi_{n-1, m+1}\right|^{2}=E_{n m}-K_{n m}$ by the eigenvalue relation (69). Similarly, considering the norm of $A_{n m} \psi_{n m}$ gives an identical value $\left|A_{n m} \psi_{n m}\right|^{2}=E_{n m}-K_{n m}$.

Specifically, to construct the normalized shift operators, let

$$
\begin{aligned}
& \mathcal{A}_{n m}^{\dagger}=\sigma_{n m} c_{n m} A_{n m}^{\dagger} \\
& \mathcal{A}_{n m}=\sigma_{n m}^{\prime} c_{n m}^{\prime} A_{n m},
\end{aligned}
$$

where $\sigma_{n m}$ and $\sigma_{n m}^{\prime}$ represent the phases, and $c_{n m}$ and $c_{n m}^{\prime}$ represent the normalization factors. Thus, insisting that both $\psi_{n m}=\mathcal{A}_{n m}^{\dagger} \psi_{n-1, m+1}$ and $\psi_{n-1, m+1}=\mathcal{A}_{n m} \psi_{n m}$ be normalized gives $c_{n m}=c_{n m}^{\prime}=\left(E_{n m}-K_{n m}\right)^{-1 / 2}$.

The phases (signs) of the solutions to an eigenproblem (66) or (70) are not determined by the eigenproblem itself, but rather are a matter of convention. For instance, it may be chosen that the wave functions are positive as $r \rightarrow 0$ or positive as $r \rightarrow \infty$. Thus, the phases $\sigma_{n m}$ and $\sigma_{n m}^{\prime}$ appearing in the normalized shift operators cannot be deduced purely from the factorization formalism but must rather be chosen so that the shift operators yield wave functions with phases consistent with the adopted convention. The self-consistency requirement that $\psi_{n m}=\mathcal{A}_{n m}^{\dagger} \psi_{n-1, m+1}=\mathcal{A}_{n m}^{\dagger} \mathcal{A}_{n m} \psi_{n m}$ imposes the constraint that $\sigma_{n m}=$ $\sigma_{n m}^{\prime}$.

If the normalized shift operators are known for all $m$, then we have a way of expressing eigenfunctions of any Hamiltonian $H_{m+k}$ in terms of those of $H_{m}$, via (77) or (78). In particular, if $k$ is a positive integer, then we repeatedly apply $\mathcal{A}$ to obtain

$$
\psi_{n, m+k}=\mathcal{A}_{n+1, m+k-1} \mathcal{A}_{n+2, m+k-2} \cdots \mathcal{A}_{n+k, m} \psi_{n+k, m}
$$

\section{B. Shift operators for Laguerre functions}

We now deduce shift operators on the $l$ quantum number of the Laguerre radial functions

$S_{n l}(r)$. We deduce these shift operators by recasting the differential equation (16) into the 
form of a factorizable $n$-dependent Hamiltonian eigenproblem, so that we may use the formalism laid out in Sec. IV A. Specifically, (16) can be rewritten in terms of the Hamiltonian operator (54) as

$$
H_{n l} S_{n l}(r)=-1 S_{n l}(r)
$$

where $E_{n l}=-1$ for all $n$ and $l$. However, to put this Hamiltonian into one of the standard factorizable forms cataloged by Infeld and Hull [29], we must first eliminate the firstderivative term, through a similarity transformation $r^{1 / 2} H_{n l} r^{-1 / 2}$. The Hamiltonian equation (83) becomes

$$
\left[-\frac{d^{2}}{d r^{2}}+\frac{\left(l+\frac{1}{2}\right)\left(l+\frac{3}{2}\right)}{r^{2}}-\frac{2 \alpha_{n l}}{r}\right] R_{n l}(r)=-1 R_{n l}(r)
$$

where

$$
R_{n l}(r)=r^{1 / 2} S_{n l}(r)
$$

Comparing (84) with the standard form for Coulomb-type problems, given by (8.0.1) of Ref. [29], yields the factorization [55]

$$
\tilde{A}_{n l}^{\dagger}(r)=\left(-\frac{d}{d r}-\frac{l+\frac{3}{2}}{r}+\frac{\alpha_{n l}}{l+\frac{3}{2}}\right) \quad \tilde{A}_{n l}(r)=\left(\frac{d}{d r}-\frac{l+\frac{3}{2}}{r}+\frac{\alpha_{n l}}{l+\frac{3}{2}}\right)
$$

and constant term

$$
K_{n l}=-\frac{\alpha_{n l}^{2}}{\left(l+\frac{3}{2}\right)^{2}} .
$$

The tildes on $\tilde{A}^{\dagger}$ and $\tilde{A}$ indicate that these are the shift operators corresponding to the similarity transformed Hamiltonian operator (84). We also note that $K_{0 l}=-1=E_{0 l}$ $(l=0,1, \ldots)$, thus satisfying the condition to obtain the relationships (67)-69) between partner Hamiltonian eigenfunctions and eigenvalues, equivalent to $\tilde{A}_{0 l} R_{0 l}=0$ and thus $A_{0 l} S_{0 l}=0$ (see Sec. IV A).

From 86 and (87), we can write 84 in the $n$-dependent factorized form of (71), as

$$
\left[\left(-\frac{d}{d r}-\frac{l+\frac{3}{2}}{r}+\frac{\alpha_{n l}}{l+\frac{3}{2}}\right)\left(\frac{d}{d r}-\frac{l+\frac{3}{2}}{r}+\frac{\alpha_{n l}}{l+\frac{3}{2}}\right)-\frac{\alpha_{n l}^{2}}{\left(l+\frac{3}{2}\right)^{2}}\right] R_{n l}(r)=-1 R_{n l}(r) .
$$

The normalized shift operators 80 - 81 for the functions $R_{n l}(r)$ are given by

$$
\begin{aligned}
& \tilde{\mathcal{A}}_{n l}^{\dagger}(r)=\frac{-\left(l+\frac{3}{2}\right)}{\sqrt{n(n+2 l+3)}}\left(-\frac{d}{d r}-\frac{l+\frac{3}{2}}{r}+\frac{\alpha_{n l}}{l+\frac{3}{2}}\right) \\
& \tilde{\mathcal{A}}_{n l}(r)=\frac{-\left(l+\frac{3}{2}\right)}{\sqrt{n(n+2 l+3)}}\left(\frac{d}{d r}-\frac{l+\frac{3}{2}}{r}+\frac{\alpha_{n l}}{l+\frac{3}{2}}\right) .
\end{aligned}
$$


Here we adopt phases $\sigma_{n l}=-1$, to enforce the convention that $R_{n l}(r)$ [and thus $S_{n l}(r)$ below] is always positive at the origin.

To obtain the shift operators for the functions $S_{n l}(r)$, we must apply the inverse similarity transformation. Thus, $\mathcal{A}_{n l}^{\dagger}(r)=r^{-1 / 2} \tilde{\mathcal{A}}_{n l}^{\dagger}(r) r^{1 / 2}$ gives

$$
\mathcal{A}_{n l}^{\dagger}(r)=\frac{-\left(l+\frac{3}{2}\right)}{\sqrt{n(n+2 l+3)}}\left(-\frac{d}{d r}-\frac{l+2}{r}+\frac{\alpha_{n l}}{l+\frac{3}{2}}\right),
$$

and, similarly, $\mathcal{A}_{n l}(r)=r^{-1 / 2} \tilde{\mathcal{A}}_{n l}(r) r^{1 / 2}$ gives

$$
\mathcal{A}_{n l}(r)=\frac{-\left(l+\frac{3}{2}\right)}{\sqrt{n(n+2 l+3)}}\left(\frac{d}{d r}-\frac{l+1}{r}+\frac{\alpha_{n l}}{l+\frac{3}{2}}\right) .
$$

\section{Deriving the action of radial operators across irreps using the shift operators}

Now that we have shift operators relating $S_{n l}(r)$ functions with different $l$,

$$
S_{n, l+1}(r)=\mathcal{A}_{n+1, l}(r) S_{n+1, l}(r) \quad S_{n l}(r)=\mathcal{A}_{n l}^{\dagger}(r) S_{n-1, l+1}(r)
$$

we can derive expressions for the matrix elements between functions $S_{n l}$ and $S_{n^{\prime} l^{\prime}}$ for $l^{\prime}=l \pm k$, for $k=1,2, \ldots$, by writing $S_{n^{\prime}, l+k}$ in terms of $S_{n l}$ using the relationships (93). Note that the matrix elements for $l^{\prime}=l-k$ can be obtained from the matrix elements of $l^{\prime}=l+k$ by making the appropriate substitutions (Appendix A), and thus it is sufficient to consider only $l^{\prime}=l+k$.

For $l^{\prime}=l \pm 1$, we simply apply the raising shift operator to $S_{n+1, l}$

$$
\begin{aligned}
S_{n, l+1}(r) & =\mathcal{A}_{n+1, l}(r) S_{n+1, l}(r) \\
& =\frac{-\left(l+\frac{3}{2}\right)}{\sqrt{(n+1)(n+2 l+4)}}\left(\frac{d}{d r}-\frac{l+1}{r}+\frac{\alpha_{n l}}{\alpha_{0 l}}\right) S_{n+1, l}(r) .
\end{aligned}
$$

Applying (51) and 52 to (94),

$$
\begin{aligned}
S_{n, l+1}(r)=\frac{-\left(l+\frac{3}{2}\right)}{\sqrt{(n+1)(n+2 l+4)}}\left[\frac{n+1}{l+\frac{3}{2}}\right. & S_{n+1, l}(r) \\
& \left.\quad-2 \sum_{i=0}^{n}\left(\frac{(n+1) !(i+2 l+2) !}{i !(n+2 l+3) !}\right)^{1 / 2} S_{i l}(r)\right] .
\end{aligned}
$$

For $l^{\prime}=l+2$, the raising shift operator must be applied twice,

$$
S_{n, l+2}(r)=\mathcal{A}_{n+1, l+1}(r) S_{n+1, l+1}(r)=\mathcal{A}_{n+1, l+1}(r) \mathcal{A}_{n+2, l}(r) S_{n+2, l}(r) .
$$


From this we have that

$$
S_{n, l+2}(r)=\sqrt{\frac{(n+1)(n+2)}{(n+2 l+6)(n+2 l+5)}} S_{n+2, l}(r)+\sum_{i=0}^{n+1} c_{i} \sqrt{\frac{n !(i+2 l+2) !}{i !(n+2 l+6) !}} S_{i l}(r),
$$

where

$$
c_{i}=(2 l+4)(2 l+3) n-(2 l+5)(2 l+4) i+(2 l+4)(2 l+3) .
$$

Similarly, we could obtain the explicit relationship between $S_{n^{\prime}, l+k}$ and $S_{n l}$ for any $k$ by applying the shift operator to $S_{n-k, l} k$ times. However, we only explicitly calculate examples of matrix elements for $l^{\prime}=l \pm 1$ and $l^{\prime}=l \pm 2$ in this paper.

Once we have an expression for $S_{n, l+k}(r)$ in terms of $S_{n l}(r)$, we can then use the matrix elements already calculated for $l=l^{\prime}$ to derive the matrix elements for $l^{\prime} \neq l$. For example,

$$
\begin{aligned}
r S_{n, l+1}(r)=\frac{-\left(l+\frac{3}{2}\right)}{\sqrt{(n+1)(n+2 l+4)}}\left[\frac{n+1}{l+\frac{3}{2}} r S_{n+1, l}(r)\right. & \\
& \left.\quad-2 \sum_{i=0}^{n}\left(\frac{(n+1) !(i+2 l+2) !}{i !(n+2 l+3) !}\right)^{1 / 2} r S_{i l}(r)\right] .
\end{aligned}
$$

From (46), we then deduce that

$$
\begin{array}{r}
r S_{n, l+1}(r)=-\frac{1}{2} \sqrt{(n+1)(n+2)} S_{n+2, l}+\sqrt{(n+1)(n+2 l+4)} S_{n+1, l}(r) \\
-\frac{1}{2} \sqrt{(n+2 l+3)(n+2 l+4)} S_{n l}(r) .
\end{array}
$$

Using the orthonormality of the $S_{n l}(r)$ we can read off the matrix elements $\left\langle n^{\prime} l|r| n, l+1\right\rangle$ from this expression, i.e.,

$$
\begin{aligned}
\langle n+2, l|r| n, l+1\rangle & =-\frac{1}{2} \sqrt{(n+1)(n+2)} \\
\langle n+1, l|r| n, l+1\rangle & =\sqrt{(n+1)(n+2 l+4)} \\
\langle n l|r| n, l+1\rangle & =-\frac{1}{2} \sqrt{(n+2 l+3)(n+2 l+4)}
\end{aligned}
$$

and all other matrix elements are zero. Matrix elements of select operators between functions with $l^{\prime}=l \pm 1$ or $l^{\prime}=l \pm 2$ are given in Appendix A (Tables II and III).

\section{MATRIX ELEMENTS IN THE LAGUERRE FUNCTION BASIS}

Now that we have a way to calculate matrix elements of radial operators with respect to the $S_{n l}$ basis on $\mathbb{R}^{+}$, we can calculate the matrix elements for irreducible tensor operators 
in the $\Lambda_{n l m}$ basis on $\mathbb{R}^{3}$. In Sec. II A we saw that the matrix element for $\mathcal{O}_{a \alpha}=R \Theta_{a \alpha}$ can be written $(20)$ as

$$
\left\langle n^{\prime} l^{\prime} m^{\prime}\left|\mathcal{O}_{a \alpha}\right| n l m\right\rangle=\left\langle n^{\prime} l^{\prime}|\gamma(R)| n l\right\rangle\left\langle l^{\prime} m^{\prime}\left|\Theta_{a \alpha}\right| l m\right\rangle
$$

where $\gamma(R)$ is given by (21). From standard angular momentum theory for spherical tensor operators [19], matrix elements involving different angular momentum projection quantum numbers $m$ and $m^{\prime}$ are all related via the Wigner-Eckart theorem, so we need only consider the reduced matrix elements. The reduced matrix element of $\mathcal{O}_{a \alpha}$ is given by the product of the $\mathrm{SO}(3)$ reduced matrix element of the angular operator and the matrix element of the radial part in the $S_{n l}$ basis,

$$
\left\langle n^{\prime} l^{\prime}|| \mathcal{O}_{a}|| n l\right\rangle=\left\langle n^{\prime} l^{\prime}|\gamma(R)| n l\right\rangle\left\langle l^{\prime} \| \Theta_{a}|| l\right\rangle
$$

Note that we follow the normalization and phase convention of, e.g., Refs. [19, 56] for the reduced matrix elements in the Wigner-Eckart theorem, i.e.,

$$
\left\langle l^{\prime} m^{\prime}\left|\Theta_{a \alpha}\right| l m\right\rangle=\hat{l}^{-1}\left(l m ; a \alpha \mid l^{\prime} m^{\prime}\right)\left\langle l^{\prime}|| \Theta_{a}|| l\right\rangle
$$

where the quantity delimited by parentheses is a Clebsch-Gordan coefficient, and $\hat{l}=$ $\sqrt{2 l+1}$.

To illustrate this method of calculating the matrix elements in the $\Lambda_{n l m}(\mathbf{r})$ basis, we will consider several examples, specifically, $r^{k}$ for $k \in \mathbb{Z}, \nabla^{2}$ and $\nabla_{1}$.

\section{A. Matrix elements of the purely radial operator $r^{k}$}

Matrix elements of polynomial operators that depend only on $r$ are particularly simple to calculate. In spherical tensor form, $r^{k} \equiv r^{k} \mathbf{1}_{00}$. Applying the factorization in (104), we can write

$$
\left\langle n^{\prime} l^{\prime}|| r^{k} \mathbf{1}_{00}|| n l\right\rangle=\left\langle n^{\prime} l^{\prime}\left|\gamma\left(r^{k}\right)\right| n l\right\rangle\left\langle l^{\prime}|| \mathbf{1}_{00}|| l\right\rangle
$$

The reduced matrix element of the identity operator is $\left\langle l^{\prime} \| \mathbf{1}_{00}|| l\right\rangle=\hat{l} \delta_{l^{\prime} l}$, and, under the transformation (21), $\gamma\left(r^{k}\right)=r^{k}$ for any $k$. Therefore, 106 reduces to

$$
\left\langle n^{\prime} l^{\prime}|| r^{k} \mathbf{1}_{00}|| n l\right\rangle=\left\langle n^{\prime} l\left|r^{k}\right| n l\right\rangle \hat{l} .
$$

The radial matrix elements are already known from Sec. IIIC and can be obtained from Table \for select values of $k$. 


\section{B. Matrix elements of the scalar operator $\nabla^{2}$}

The Laplacian $\nabla^{2}$ has the well known form [19]

$$
\nabla^{2}=\frac{1}{r^{2}} \frac{\partial}{\partial r} r^{2} \frac{\partial}{\partial r}-\frac{L^{2}}{r^{2}}
$$

We factor each of the terms contributing to the matrix element of this operator into radial and angular parts, according to 104, and recall $L^{2}|l m\rangle=l(l+1)|l m\rangle$, yielding

$$
\begin{aligned}
& \left\langle n^{\prime} l^{\prime}\left\|\nabla^{2}\right\| n l\right\rangle \\
& =\left\langle n^{\prime} l\left|\gamma\left(r^{-2} \frac{d}{d r} r^{2} \frac{d}{d r}\right)\right| n l\right\rangle \hat{l} \delta_{l l^{\prime}}-\left\langle n^{\prime} l^{\prime}\left|\gamma\left(r^{-2}\right)\right| n l\right\rangle\left\langle l^{\prime}\left\|L^{2}\right\| l\right\rangle \\
& =\left\langle n^{\prime} l\left|\frac{d^{2}}{d r^{2}}\right| n l\right\rangle \hat{l}-\left\langle n^{\prime} l\left|r^{-2}\right| n l\right\rangle l(l+1) \hat{l}
\end{aligned}
$$

The matrix elements of $d^{2} / d r^{2}$ and $r^{-2}$ can be read from Table I, and we find that

$$
\left\langle n^{\prime} l\left\|\nabla^{2}\right\| n l\right\rangle= \begin{cases}-\frac{4 n+2 l+3}{2 l+3} \hat{l} & n^{\prime}=n \\ \frac{4 n^{\prime}+4 l+6}{2 l+3} \hat{l}\left(\frac{n !\left(n^{\prime}+2 l+2\right) !}{n^{\prime} !(n+2 l+2) !}\right)^{1 / 2} & n^{\prime} \leq n-1 \\ \frac{4 n+4 l+6}{2 l+3} \hat{l}\left(\frac{n^{\prime} !(n+2 l+2) !}{n !\left(n^{\prime}+2 l+2\right) !}\right)^{1 / 2} & n^{\prime} \geq n+1 .\end{cases}
$$

\section{Matrix elements of the tensor operator $\nabla_{1}$}

We now want to calculate the reduced matrix elements the gradient operator $\boldsymbol{\nabla}$, which can be expressed as a rank 1 tensor $\nabla_{1}$ with components [19]

$$
\nabla_{1 \mu}=\left(\frac{4 \pi}{3}\right)^{\frac{1}{2}}\left\{Y_{1 \mu} \frac{\partial}{\partial r}-\frac{\sqrt{2}}{r}\left[Y_{1} \otimes L_{1}\right]_{1 \mu}\right\}
$$

where $Y_{1}$ is the irreducible tensor whose components are the spherical harmonics $Y_{1 \mu}(\hat{r})$, and $L_{1}$ is the orbital angular momentum operator, likewise considered as a spherical tensor. The reduced matrix element of $\nabla_{1}$ is thus given by

$$
\left\langle n^{\prime} l^{\prime}\left\|\nabla_{1}\right\| n l\right\rangle=\left(\frac{4 \pi}{3}\right)^{\frac{1}{2}}\left\langle n^{\prime} l^{\prime}\left\|\left[Y_{1} \frac{\partial}{\partial r}-\frac{\sqrt{2}}{r}\left[Y_{1} \otimes L_{1}\right]_{1}\right]\right\| n l\right\rangle .
$$

In each term, the radial and angular functions can be separated by applying (104). The angular part of the second term can be factored as

$$
\begin{aligned}
\left\langle n^{\prime} l^{\prime}\left\|\left[Y_{1} \otimes L_{1}\right]_{1}\right\| n l\right\rangle=(-1)^{l+l^{\prime}-1} \sqrt{\frac{3}{2 l^{\prime}+1}} \sum_{l_{1}}\left\{\begin{array}{ccc}
1 & 1 & 1 \\
l & l^{\prime} & l_{1}
\end{array}\right\} \\
\\
\times\left\langle n^{\prime} l^{\prime}\left\|Y_{1}\right\| n_{1} l_{1}\right\rangle\left\langle n_{1} l_{1}\left\|L_{1}\right\| n l\right\rangle
\end{aligned}
$$


by the standard reduction formula for reduced matrix elements of product operators on a single space [19]. Using the reduced matrix elements of $Y_{1}$ and $L_{1}$ given in [19], the expression for the reduced matrix element of $\nabla_{1}$ becomes

$$
\begin{aligned}
\left\langle n^{\prime} l^{\prime}|| \nabla_{1} \| n l\right\rangle= & \sqrt{\frac{l+1}{2 l+3}}\left\langle n^{\prime} l^{\prime}\left|\left(\frac{d}{d r}-\frac{l+1}{r}\right)\right| n l\right\rangle \delta_{l^{\prime}, l+1} \\
& -\sqrt{\frac{l}{2 l-1}}\left\langle n^{\prime} l^{\prime}\left|\left(\frac{d}{d r}+\frac{l}{r}\right)\right| n l\right\rangle \delta_{l^{\prime}, l-1} .
\end{aligned}
$$

The terms contributing to the radial matrix elements can then be read from Table II] and we have

$$
\left\langle n^{\prime}, l+1\left\|\nabla_{1}\right\| n l\right\rangle= \begin{cases}-2\left(n^{\prime}+l+3 / 2\right)\left(\frac{l+1}{2 l+3}\right)^{\frac{1}{2}}\left(\frac{n^{\prime} !(n+2 l+2) !}{n !\left(n^{\prime}+2 l+4\right) !}\right)^{\frac{1}{2}} & n^{\prime} \geq n \\ \left(\frac{n(l+1)}{(2 l+3)(n+2 l+3)}\right)^{1 / 2} & n^{\prime}=n-1 \\ 0 & n^{\prime} \leq n-2 .\end{cases}
$$

and

$$
\left\langle n^{\prime}, l-1|| \nabla_{1}|| n l\right\rangle= \begin{cases}0 & n^{\prime} \geq n+2 \\ -\left(\frac{(n+1) l}{(2 l-1)(n+2 l+2)}\right)^{1 / 2} & n^{\prime}=n+1 \\ -2\left(n^{\prime}+l+1 / 2\right)\left(\frac{l}{2 l-1}\right)^{\frac{1}{2}}\left(\frac{n !\left(n^{\prime}+2 l\right) !}{n^{\prime} !(n+2 l+2) !}\right)^{\frac{1}{2}} & n^{\prime} \leq n .\end{cases}
$$

\section{CONCLUSION}

It has long been recognized that an $\mathrm{SU}(1,1) \times \mathrm{SO}(3)$ algebraic structure, together with shift operators between $\mathrm{SU}(1,1)$ irreps, form a successful combination in the treatment of the classic sets of central force eigenfunctions - those of the harmonic oscillator and Coulomb problems. We have now demonstrated that a similar framework can be developed and effectively applied to the Laguerre functions, a basis set of practical value in quantum mechanical one-body, few-body, and many-body problems.

The essential results thus obtained provide for the evaluation of matrix elements of spherical tensor operators. This is the immediate application of interest in formulating quantum mechanical problems for numerical solution. The evaluation of matrix elements is accomplished by decomposing the full operator, in the three-dimensional coordinate space, into parts which act only radial or angular coordinates. Then, recall the structure outlined in 
Fig. 1: (1) the $\mathrm{SU}(1,1)$ algebra of the radial functions is used to deduce radial matrix elements, while (2) the shift operators connect the different families of radial functions, or $\mathrm{SU}(1,1)$ irreps, associated with different angular functions, and (3) the $\mathrm{SO}(3)$ angular momentum algebra is used to address the action of the operator on the angular functions themselves. The $\mathrm{SU}(1,1)$ algebra follows directly from the eigenvalue equation satified by the radial functions. The ladder operators are obtained by factorization (or, equivalently, SUSY QM) methods, reformulated to allow for quantum number dependence of the shift operators. And the $\mathrm{SO}(3)$ algebra provides the familiar tools of the Wigner-Eckart theorem and Racah's reduction formulas for the angular operators.

Although the results, as presented, have been specialized to the Laguerre functions on $\mathbb{R}^{3}$, their applicability is broader. In particular, the results for the radial functions as a basis on $\mathbb{R}^{+}$stand alone or in conjunction with any decomposition of $N$-dimensional coordinates on $\mathbb{R}^{N}$ into a radial coordinate on $\mathbb{R}^{+}$and angular coordinates on the $(N-1)$-sphere $S^{N-1}$. For instance, functions of the Laguerre type considered here are applied as hyperradial basis functions in few-body calculations in Jacobi coordinates (e.g., Ref. [57]). Alternatively, much as the $\mathrm{SU}(1,1)$ treatment of the harmonic oscillator radial functions may be extended to a broader family of modified harmonic oscillator functions, by transitioning from a discrete $\mathrm{SU}(1,1)$ irrep label $t=l+3 / 2$ to a continuous label [23] (physically corresponding from the transition from a pure oscillator potential to a Davidson potential [58], containing an additional $1 / r^{2}$ dependence), a similar generalization may be carried out for the present results, yielding an algebraic description of families of modified Laguerre functions. The general framework may also be carried over to the Coulomb-Sturmian functions, for which the $\mathrm{SU}(1,1)$ radial algebra is considered in Ref. [59].

Finally, it is worth noting that, so far, we have focused exclusively on the $\mathrm{SU}(1,1)$ character of the basis functions, rather than on the $\mathrm{SU}(1,1)$ tensorial properties of operators on the radial space. Through these properties, it is possible to benefit from the Wigner-Eckart theorem, not only of the $\mathrm{SO}(3)$ angular algebra as we have done so far, but also of the $\mathrm{SU}(1,1)$ radial algebra. Matrix elements involving different members of the same irrep (i.e., different radial functions for the same angular momentum) may then be related to each other through $\mathrm{SU}(1,1)$ Clebsch-Gordan coefficients. The SU(1,1) Wigner-Eckhart theorem, applied in this fashion, has been a powerful tool in the construction of closed-form expressions for matrix elements in the classic harmonic oscillator and Coulomb eigenproblems [20, 60]. 


\section{Acknowledgements}

We would like to thank C. Kolda and J. de Blas for valuable discussions, M. Wolf for comments on the manuscript and A. I. Rakoski for verification of expressions for matrix elements. This work was supported by the Research Corporation for Science Advancement under a Cottrell Scholar Award and by the US Department of Energy under Grant No. DEFG02-95ER-40934. 


\section{Appendix A: Matrix elements between Laguerre radial functions}

In the following tables, we have summarized the matrix elements of some of the most basic radial operators that can be constructed from $r, r^{-1}$ and $d / d r$, taken in the Laguerre function radial basis. Matrix elements within a single $\mathrm{SU}(1,1)$ irrep, i.e., between radial functions $S_{n l}$ of the same $l$, are given in Table I. Matrix elements between radial functions belonging to different $\mathrm{SU}(1,1)$ irreps, corresponding to angular momenta differing by $\Delta l=1$ and 2, are given in Tables II] and [II], respectively.

Note that the matrix elements in Tables IIIII are of the form $\left\langle n^{\prime} l|R| n l\right\rangle,\left\langle n^{\prime} l|R| n, l+1\right\rangle$, or $\left\langle n^{\prime} l|R| n, l+2\right\rangle$, respectively, as this is the form that is most naturally derived from applying the shift operators (see Sec. IV). However, one may express the l-changing matrix elements in the form $\left\langle n^{\prime}, l-1|R| n l\right\rangle$ or $\left\langle n^{\prime}, l-2|R| n l\right\rangle$ by making the appropriate substitution. For instance, to obtain $\left\langle n^{\prime}, l-1|r| n l\right\rangle$ from the matrix elements in Table II, one may simply make the substitution $l \rightarrow l-1$. Alternatively, matrix elements of the form $\left\langle n^{\prime}, l-1|R| n l\right\rangle$ or $\left\langle n^{\prime}, l-2|R| n l\right\rangle$ may be obtained by Hermitian conjugation. For instance, to obtain $\left\langle n^{\prime}, l-1|r| n l\right\rangle$ from the matrix elements in Table II. one may simply note that $\left\langle n^{\prime}, l+1|r| n l\right\rangle=\left\langle n l|r| n^{\prime}, l+1\right\rangle$, since the matrix elements are real-valued, and make the interchange $n \leftrightarrow n^{\prime}$. When applying Hermitian conjugation to differential operators appearing in Tables IIIII, recall that the operator $d / d r$ is anti-Hermitian (e.g., Ref. [16]).

TABLE I: Matrix elements between functions in the $S_{n l}$ basis.

$$
\begin{array}{rlr}
\left\langle n^{\prime} l|r| n l\right\rangle & n^{\prime} \geq n+2 & 0 \\
n^{\prime} & =n+1 & -\frac{1}{2}((n+1)(n+2 l+3))^{1 / 2} \\
n^{\prime} & =n & n+l+\frac{3}{2} \\
& n^{\prime}=n-1 & -\frac{1}{2}(n(n+2 l+2))^{1 / 2} \\
& n^{\prime} \leq n-2 & 0 \\
\left.\hline n^{\prime} l\left|r^{2}\right| n l\right\rangle & n^{\prime} \geq n+3 & 0
\end{array}
$$


TABLE I -continued from previous page

\begin{tabular}{|c|c|c|}
\hline \multicolumn{2}{|c|}{$n^{\prime}=n+2$} & $\frac{1}{4}((n+1)(n+2)(n+2 l+3)(n+2 l+4))^{1 / 2}$ \\
\hline & $n^{\prime}=n+1$ & $-(n+l+2)((n+1)(n+2 l+3))^{1 / 2}$ \\
\hline & $n^{\prime}=n$ & $\left(n+l+\frac{3}{2}\right)(n+l+2)+\frac{1}{2} n(n+2 l+2)$ \\
\hline & $n^{\prime}=n-1$ & $-(n+l+1)(n(n+2 l+2))^{1 / 2}$ \\
\hline & $n^{\prime}=n-2$ & $\frac{1}{4}(n(n-1)(n+2 l+2)(n+2 l+1))^{1 / 2}$ \\
\hline & $n^{\prime} \leq n-3$ & 0 \\
\hline$\left\langle n^{\prime} l\left|\frac{1}{r}\right| n l\right\rangle$ & $n^{\prime} \geq n$ & $\frac{1}{l+1}\left(\frac{n^{\prime} !(n+2 l+2) !}{n !\left(n^{\prime}+2 l+2\right) !}\right)^{1 / 2}$ \\
\hline & $n^{\prime} \leq n$ & $\frac{1}{l+1}\left(\frac{n !\left(n^{\prime}+2 l+2\right) !}{n^{\prime} !(n+2 l+2) !}\right)^{1 / 2}$ \\
\hline$\left\langle n^{\prime} l\left|\frac{1}{r^{2}}\right| n l\right\rangle$ & $n^{\prime} \geq n$ & $\left(\frac{2 n^{\prime}(2 l+3)-2 n(2 l+1)+4 l+6}{(l+1)(2 l+1)(2 l+3)}\right)\left(\frac{n^{\prime} !(n+2 l+2) !}{n !\left(n^{\prime}+2 l+2\right) !}\right)^{1 / 2}$ \\
\hline & $n^{\prime} \leq n$ & $\left(\frac{2 n(2 l+3)-2 n^{\prime}(2 l+1)+4 l+6}{(l+1)(2 l+1)(2 l+3)}\right)\left(\frac{n !\left(n^{\prime}+2 l+2\right) !}{n^{\prime} !(n+2 l+2) !}\right)^{1 / 2}$ \\
\hline$\left\langle n^{\prime} l\left|r \frac{d}{d r}\right| n l\right\rangle$ & $n^{\prime} \geq n+2$ & 0 \\
\hline & $n^{\prime}=n+1$ & $\frac{1}{2}((n+1)(n+2 l+3))^{1 / 2}$ \\
\hline & $n^{\prime}=n$ & $-\frac{1}{2}$ \\
\hline & $n^{\prime}=n-1$ & $-\frac{1}{2}(n(n+2 l+2))^{1 / 2}$ \\
\hline & $n^{\prime} \leq n-2$ & 0 \\
\hline$\left\langle n^{\prime} l\left|\frac{d}{d r}\right| n l\right\rangle$ & $n^{\prime} \geq n+1$ & $\left(\frac{n^{\prime} !(n+2 l+2) !}{n !\left(n^{\prime}+2 l+2\right) !}\right)^{1 / 2}$ \\
\hline & $n^{\prime}=n$ & 0 \\
\hline & $n^{\prime} \leq n-1$ & $-\left(\frac{n !\left(n^{\prime}+2 l+2\right) !}{n^{\prime} !(n+2 l+2) !}\right)^{1 / 2}$ \\
\hline
\end{tabular}


TABLE I -continued from previous page

$$
\begin{array}{rlr}
\left\langle n^{\prime} l\left|\frac{d^{2}}{d r^{2}}\right| n l\right\rangle & n^{\prime} \geq n+1 & -\frac{(2 l+4)(2 l+1) n-2 l(2 l+3) n^{\prime}+(2 l+3)(2 l+2)}{(2 l+3)(2 l+1)}\left(\frac{n^{\prime} !(n+2 l+2) !}{n !\left(n^{\prime}+2 l+2\right) !}\right)^{1 / 2} \\
n^{\prime} & =n & -\frac{4 n(l+1)+2 l+3}{(2 l+3)(2 l+1)} \\
n^{\prime} \leq n-1 & -\frac{(2 l+4)(2 l+1) n^{\prime}-2 l(2 l+3) n+(2 l+3)(2 l+2)}{(2 l+3)(2 l+1)}\left(\frac{n !\left(n^{\prime}+2 l+2\right) !}{n^{\prime} !(n+2 l+2) !}\right)^{1 / 2} \\
\hline
\end{array}
$$

\begin{tabular}{|c|c|c|}
\hline \multirow[t]{3}{*}{$\left\langle n^{\prime} l \mid n, l+1\right\rangle$} & $n^{\prime} \geq n+2$ & 0 \\
\hline & $n^{\prime}=n+1$ & $-\left(\frac{n+1}{n+2 l+4}\right)^{1 / 2}$ \\
\hline & $n^{\prime} \leq n$ & $(2 l+3)\left(\frac{n !\left(n^{\prime}+2 l+2\right) !}{n^{\prime} !(n+2 l+4) !}\right)^{1 / 2}$ \\
\hline \multirow[t]{5}{*}{$\left\langle n^{\prime} l|r| n, l+1\right\rangle$} & $n^{\prime} \geq n+3$ & 0 \\
\hline & $n^{\prime}=n+2$ & $\frac{1}{2}((n+1)(n+2))^{1 / 2}$ \\
\hline & $n^{\prime}=n+1$ & $-((n+1)(n+2 l+4))^{1 / 2}$ \\
\hline & $n^{\prime}=n$ & $\frac{1}{2}((n+2 l+4)(n+2 l+3))^{1 / 2}$ \\
\hline & $n^{\prime} \leq n-1$ & 0 \\
\hline \multirow[t]{2}{*}{$\left\langle n^{\prime} l\left|\frac{1}{r}\right| n, l+1\right\rangle$} & $n^{\prime} \geq n+1$ & 0 \\
\hline & $n^{\prime} \leq n$ & $\left(2(n+1)-2 n^{\prime}\right)\left(\frac{n !\left(n^{\prime}+2 l+2\right) !}{n^{\prime} !(n+2 l+4) !}\right)^{1 / 2}$ \\
\hline \multirow[t]{2}{*}{$\left\langle n^{\prime} l\left|\frac{d}{d r}\right| n, l+1\right\rangle$} & $n^{\prime} \geq n+2$ & 0 \\
\hline & $n^{\prime}=n+1$ & $\left(\frac{n+1}{n+2 l+4}\right)^{1 / 2}$ \\
\hline
\end{tabular}

TABLE II: Matrix elements between functions in the $S_{n, l+1}$ and $S_{n l}$ bases. 
TABLE II -continued from previous page

$n^{\prime} \leq n \quad\left(n^{\prime}(2 l+4)-n(2 l+2)+1\right)\left(\frac{n !\left(n^{\prime}+2 l+2\right) !}{n^{\prime} !(n+2 l+4) !}\right)^{1 / 2}$

TABLE III: Matrix elements between functions in the $S_{n, l+2}$ and $S_{n l}$ bases.

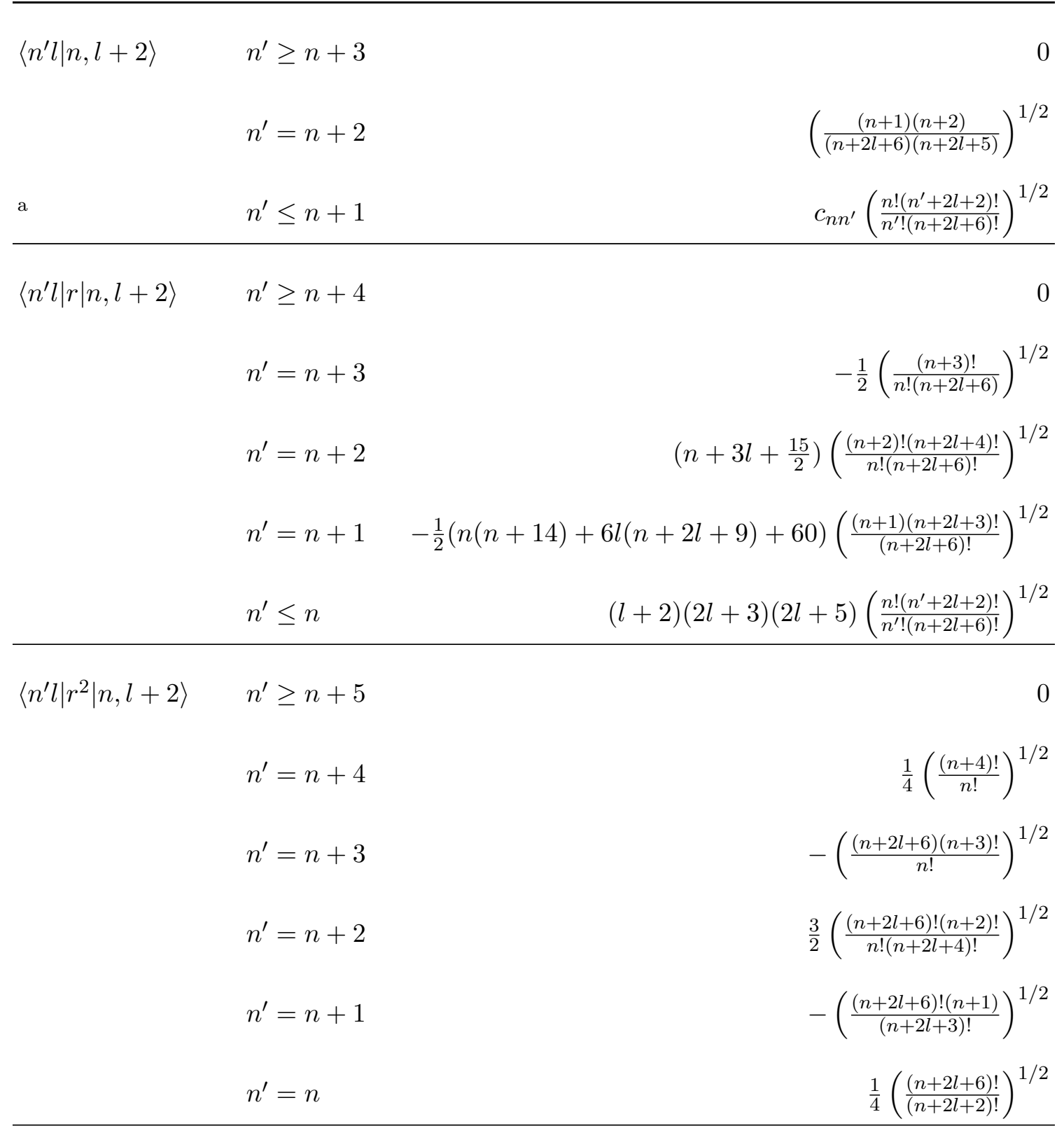


TABLE III -continued from previous page

\begin{tabular}{llr}
\hline & $n^{\prime} \leq n-1$ & 0 \\
\hline$\left\langle n^{\prime} l\left|\frac{1}{r^{2}}\right| n, l+2\right\rangle$ & $n^{\prime} \geq n+1$ & 0 \\
& $n^{\prime} \leq n$ & $\frac{2}{3}\left(n-n^{\prime}+1\right)\left(n-n^{\prime}+2\right)\left(n-n^{\prime}+3\right)\left(\frac{n !\left(n^{\prime}+2 l+2\right) !}{n^{\prime} !(n+2 l+6) !}\right)^{1 / 2}$ \\
\hline$\left\langle n^{\prime} l\left|\frac{d}{d r}\right| n, l+2\right\rangle$ & $n^{\prime} \geq n+3$ & $-\left(\frac{(n+2) !(n+2 l+4) !}{n !(n+2 l+6) !}\right)^{1 / 2}$ \\
$\mathrm{~b}$ & $n^{\prime}=n+2$ & $d_{n n^{\prime}}\left(\frac{n !\left(n^{\prime}+2 l+2\right) !}{n^{\prime} !(n+2 l+6) !}\right)^{1 / 2}$ \\
\hline$\left\langle n^{\prime} l\left|\frac{d^{2}}{d r^{2}}\right| n, l+2\right\rangle$ & $n^{\prime} \geq n+3$ & $\left(\frac{(n+2) !(n+2 l+4) !}{n !(n+2 l+6) !}\right)^{1 / 2}$ \\
& $n^{\prime}=n+2$ & $f_{n n^{\prime}}\left(\frac{n !\left(n^{\prime}+2 l+2\right) !}{n^{\prime} !(n+2 l+6) !}\right)^{1 / 2}$ \\
\hline $\mathrm{c}$ & $n^{\prime} \leq n+2$ & 0 \\
\hline
\end{tabular}

${ }^{\mathrm{a}} c_{n n^{\prime}}=(2 l+4)(2 l+3) n-(2 l+5)(2 l+4) n^{\prime}+(2 l+4)(2 l+3)$

${ }^{\mathrm{b}} d_{n n^{\prime}}=n^{\prime}\left[4 n(l+2)^{2}+l(2 l+9)+11-n^{\prime}(2 l+5)(l+3)\right]-(n+1)(2 l+3)(n(l+1)-2)$

${ }^{\text {c }} f_{n n^{\prime}}=-\frac{2}{3}\left\{n^{\prime}\left[(l+3) n^{\prime}\left((l+4) n^{\prime}-3((l+2) n+1)\right)+(l+2) n(3 n(l+1)-6)-l(l+7)-9\right]\right.$

$-n[(l+1) n(n l-9)-l(l+13)-9]+3 l\}$ 
[1] H. Shull and P.-O. Löwdin, J. Chem. Phys. 23(7), 1362 (1955).

[2] E. Filter and E. O. Steinborn, J. Math. Phys. 21, 2725 (1980).

[3] E. J. Weniger, J. Math. Phys. 26, 276 (1985).

[4] H. Shull and P.-O. Löwdin, J. Chem. Phys. 23, 1565 (1955).

[5] P. Lowdin and H. Shull, Phys. Rev. 101(6), 1730 (1956).

[6] T. Helgaker, P. Jørgensen, and J. Olsen, Molecular Electron-Structure Theory (Wiley, Chichester, 2000).

[7] S. Jacobs, M. G. Olsson, and C. Suchyta, III, Phys. Rev. D 33, 3338 (1986).

[8] L. P. Fulcher, Z. Chen, and K. C. Yeong, Phys. Rev. D 47, 4122 (1993).

[9] C. Olson and M. G. Olsson, Phys. Rev. D 49(9), 4675 (1994).

[10] M. G. Olsson and S. Veseli, Phys. Rev. D 51(9), 5079 (1995).

[11] B. D. Keister and W. N. Polyzou, J. Comput. Phys. 134, 231 (1997).

[12] M. Pervin, Semileptonic decay of heavy baryons in a constituent quark model, Ph.D. thesis, Florida State University (2005).

[13] S. Veerasamy and W. N. Polyzou, Phys. Rev. C 84, 034003 (2011).

[14] M. A. Caprio, P. Maris, and J. P. Vary, Phys. Rev. C 86, 034312 (2012).

[15] M. A. Caprio, P. Maris, and J. P. Vary, Phys. Rev. C 90, 034305 (2014).

[16] J. J. Sakurai, Modern Quantum Mechanics, rev. ed. (Addison-Wesley, Reading, Massachusetts, 1994).

[17] J. W. Negele and H. Orland, Quantum Many-Particle Systems (Addison-Wesley, Redwood City, CA, 1988).

[18] M. Moshinsky and Y. F. Smirnov, The Harmonic Oscillator in Modern Physics (Harwood Academic Publishers, Amsterdam, 1996).

[19] D. A. Varshalovich, A. N. Moskalev, and V. K. Khersonskii, Quantum Theory of Angular Momentum (World Scientific, Singapore, 1988).

[20] B. G. Wybourne, Classical Groups for Physicists (Wiley, New York, 1974).

[21] J. Č́žzek and J. Paldus, Int. J. Quantum. Chem. 12(5), 875 (1977).

[22] T. H. Cooke and J. L. Wood, Am. J. Phys. 70(9), 945 (2002).

[23] D. J. Rowe, J. Phys. A 38, 10181 (2005). 
[24] L. Armstrong Jr., Phys. Rev. A 3, 1546 (1971).

[25] B. G. Adams, J. Cizek, and J. Paldus, Adv. Quantum Chem. 19, 1 (1988).

[26] I. L. Cooper, J. Phys. A 26, 1601 (1993).

[27] E. Kelbert, A. Hyder, F. Demir, Z. T. Hlousek, and Z. Papp, J. Phys. A 40, 7721 (2007).

[28] E. Schrödinger, Proc. Roy. Irish Acad 46, 9 (1940).

[29] L. Infeld and T. E. Hull, Rev. Mod. Phys. 23, 21 (1951).

[30] F. Cooper, A. Khare, and U. Sukhatme, Phys. Rep. 251, 267 (1995).

[31] D. J. Rowe, M. J. Carvalho, and J. Repka, Rev. Mod. Phys. 84, 711 (2012).

[32] B. R. Barrett, P. Navrátil, and J. P. Vary, Prog. Part. Nucl. Phys. 69, 131 (2013).

[33] G. B. Arfken and H. J. Weber, Mathematical Methods for Physicists, 6th ed. (Academic Press, San Diego, 2005).

[34] K. T. R. Davies, S. J. Krieger, and M. Baranger, Nucl. Phys. 84, 545 (1966).

[35] B. Jonson, Phys. Rep. 389, 1 (2004).

[36] In comparing expressions in this work, such as (2), with standard treatments of the Coulomb problem (and with the electron-structure theory literature in general, e.g., Ref. [6]), one must be alert to the dichotomous use of the symbol $n$. We retain the generic notation for central force problems (e.g., Ref. [16]), in which $n$ is the radial quantum number $(n=0,1, \ldots)$, representing the number of nodes in the radial wave function. This use of $n$ is common in nuclear physics and treatments of the harmonic oscillator [18]. Then, the principal quantum number, which determines the energy degeneracies, is indicated by $N$. In particular, $N=2 n+l(N=0$, $1, \ldots)$ for the harmonic oscillator problem, with energy $E(N) \propto N+3 / 2$, or $N=n+l+1$ $(N=1,2, \ldots)$ for the Coulomb problem, with energy $E(N) \propto-1 / N^{2}$. However, in treatments of the hydrogen atom, it is the principal quantum number which is traditionally denoted by $n$. Thus, an alternative symbol, such as $\nu$, must be used for the radial quantum number, and we may write $n=\nu+l+1$, where now $E(n) \propto-1 / n^{2}$. In electron-structure theory, this hydrogenic definition of $n$ as $\nu+l+1$ in terms of the other quantum numbers $\nu$ and $l$ is conventionally carried over to other basis sets [6], such as harmonic oscillator functions and Laguerre functions, despite having no interpretation as a principal quantum number for those bases. To translate expressions from the former convention ( $n$ as radial quantum number)to the latter convention ( $n$ as hydrogenic principal quantum number), one must make replacements $n \rightarrow n-l-1$. 
[37] E. A. Hylleraas, Z. Phys. 48, 469 (1928).

[38] M. Rotenberg, Ann. Phys. (N.Y.) 19, 262 (1962).

[39] B. Klahn and W. A. Bingel, Theor. Chim. Acta 44, 27 (1977).

[40] Although the term Sturmian by itself has been used to refer to the Coulomb-Sturmian functions [38], this term is more general and may be used to refer to Sturm-Liouville expansion basis functions associated with other choices of potential $V(r)$.

[41] M. Rotenberg, Adv. At. Mol. Phys. 6, 233 (1970).

[42] E. J. Heller and H. A. Yamani, Phys. Rev. A 9, 1209 (1974).

[43] C. Coletti, D. Calderini, and V. Aquilanti, Adv. Quantum Chem. 67, 73 (2013).

[44] Z. Papp, Phys. Rev. C 38, 2457 (1988).

[45] Z. Papp and S. Moszkowski, Mod. Phys. Lett. B 22, 2201 (2008).

[46] Z. Papp, J. Phys. A 20, 153 (1987).

[47] B. Klahn and W. A. Bingel, Theor. Chim. Acta 44, 9 (1977).

[48] Although we use the term Laguerre function to refer to the full function $\Lambda_{n l m}(\mathbf{r})$ of (4), in three dimensions, as in Ref. [6], it should be noted that this term is also used in a more generic sense to refer to the corresponding unnormalized radial wave function. In particular, Ref. [33] defines the Laguerre function as $\psi_{n}^{k}(r)=r^{k / 2} L_{n}^{k}(r) e^{-r / 2}$. With the identification $k=2 l+2$, and except for the omission of the normalization factor, this may be recognized as the auxiliary radial function (that is, the radial function after absorbing the radial integration metric) $S_{n l}(r)$ associated with (4).

[49] Alternatively, in an entirely different meaning, the term Laguerre function may be used to refer to the generalization of the associated Laguerre polynomial $L_{n}^{\alpha}(x)$ to nonintegral parameters $n$ and $\alpha$ 33.

[50] Due to this similarity in functional form, the Laguerre functions have in a few instances been referred to as "Coulomb-Sturmian functions" in the hadronic and nuclear physics literature [11, 14, 15. However, it should be emphasized that the Coulomb-Sturmian functions (3) and Laguerre functions (4) are distinct, and only the former are actually obtained as solutions to the Coulomb potential's associated Sturm-Liouville problem.

[51] More precisely, the associated radial equation is of the Sturm-Liouville form $-(d / d x)\left[p(x)(d / d x) y_{n}(x)\right]+q(x) y_{n}(x)=\lambda V(x) y_{n}(x)$, with $p(x)>0$ and $q(x)>0$. If $p(x)$ and $q(x)$ are continuous and the derivative of $p(x)$ is continuous on the closed interval $[a, b]$, 
Sturm-Liouville theory then tells us that the normalized eigenfunctions form an orthonormal basis $\int_{a}^{b} y_{n}(x) y_{m}(x) V(x) d x=\delta_{m n}$ in the Hilbert Space $\mathcal{L}^{2}([a, b], V(x) d x)$, i.e., under an inner product with weighting function $V(x)$ 33].

[52] L. E. Gendenshtein, Pis'ma Zh. Eksp. Teor. Fiz. 38(6), 299 (1983).

[53] P. A. M. Dirac, The Principles of Quantum Mechanics, 4th (revised) ed., The International Series of Monographs on Physics No. 27 (Clarendon Press, Oxford, 1967).

[54] K. T. Hecht, Quantum Mechanics (Springer, Berlin, 2000).

[55] The parameter $m$ of Infeld and Hull [29] may be identified with either $l+1 / 2$ or $-(l+3 / 2)$ in the present problem (we adopt the latter choice to obtain a more convenient form for our subsequent results), and the parameter $q$ of Infeld and Hull is identified with $-\alpha_{n l}$.

[56] A. R. Edmonds, Angular Momentum in Quantum Mechanics, 2nd ed., Investigations in Physics No. 4 (Princeton University Press, Princeton, New Jersey, 1960).

[57] S. Bacca, N. Barnea, and A. Schwenk, Phys. Rev. C 86, 034321 (2012).

[58] P. M. Davidson, Proc. R. Soc. London A 135, 459 (1932).

[59] G. Lévai, B. Kónya, and Z. Papp, J. Math. Phys. 39, 5811 (1998).

[60] C. Quesne and M. Moshinsky, J. Math. Phys. 12, 1780 (1971). 